\title{
THE CLASSIFICATION OF LINKS UP TO LINK-HOMOTOPY
}

\author{
NATHAN HABEGGER AND XIAO-SONG LIN
}

Though the study of knots and links in dimension three has been with us for well over a century, progress towards the ultimate goal of their classification has been slow. Various methods have been used in their study, ranging from braid theory to the study of the link complement and its fundamental group.

Braid theory succeeded in the classification of braids (i.e., in solving the word and conjugacy problems in the braid groups). An equivalence relation generated by Markov moves on braids was found yielding the set of isotopy classes of links (see [B]). However, the combinatorics of the Markov moves are very difficult, and braid theory has not yet led to the classification of links, although recent work of Birman and Menasco shows progress in that direction [BM]. On the other hand, it has led to polynomial invariants, via the work of Jones and others [J]. (Recently, Witten [W] has given a physical interpretation of these polynomials in terms of particle "transmission." Possible relations with the results in this work are yet to be explored.)

The study of the fundamental group of the complement of a link has led to link invariants of many types. Milnor [M2] introduced higher order linking numbers, called $\bar{\mu}$-invariants, which have been shown (see [P] and [T]) to coincide with cohomological invariants [Ma] of the link complement. These invariants have proven inadequate for the classification of links, in part due to a lack of understanding of their true indeterminancy. Several years prior to the publication of [M2], Milnor introduced the notion of link-homotopy (his terminology was simply "homotopy") in [M1]. Some link-homotopy invariants of links were introduced there which turned out to be a subcollection of the $\bar{\mu}$-invariants in [M2]. It was hoped that, modulo a certain appropriate indeterminancy, these link-homotopy invariants would be able to classify links up to link-homotopy. Although this classification was achieved by Milnor for links with two and three components [M1], it was only after more than thirty years that Levine accomplished such a classification for links with four components [Le2].

The classification of links up to link-homotopy, presented here, represents a unification of the above two points of view. As such, it is a possible model for what the ultimate classification picture might look like. See [L] for a general-

Received by the editors August 30, 1989.

1980 Mathematics Subject Classification (1985 Revision). Primary 57Q35, 57Q45, 57R40; Secondary $54 \mathrm{C} 25,54 \mathrm{E} 60,55 \mathrm{Q} 25$. 
ization to $\mathbf{N}$-equivalence and [Le1] for surgery equivalence. In a forthcoming paper, [HL], we will extend the results obtained here to study the link concordance problem.

In fact, we were led to this synthesis in a somewhat awkward fashion. But in retrospect, it seems so natural that we were surprised it had not been attempted before. Closely related ideas may be found in [A], [G1], and [D].

Our results involve a mixture of topology, algebra, and combinatorics. It was the combinatorial step which went unsolved for the longest period (six months). Indeed, we even worried for a time that the combinatorial problem would turn out to be unsolvable (as is still possible for other low-dimensional combinatorial problems). Fortunately, this turned out not to be the case.

Much of the topology and corresponding algebra is not new. A key new ingredient is an understanding of the choice involved in basing a link (by which we mean the choice of a wedge of arcs connecting the base point of the link complement to the base points of the components of the link in the link complement), at least for link-homotopy. It is this choice which leads to all the interesting (though complicated) combinatorics and to the indeterminancy of the Milnor invariants. Whenever the choice of a base of a link is made, the essential part of the link, up to link-homotopy, is a pure braid with the same multiplicity as the link. Thus, we were led to study the classification of braids up to linkhomotopy. The group structure of the braid group modulo link-homotopy was already studied in the work of Goldsmith [G1]. A new result we present here is an Artin-type representation theorem for the group of link-homotopy classes of braids (see Theorem 1.7). It is at this point that Milnor's link-homotopy invariants enter into the picture.

Due to different choices of base, a link may be represented by different pure braids, up to link-homotopy. Our classification theorem states that the difference between two link-homotopically different pure braid representatives of a link can be characterized by a certain group action on the group of linkhomotopy classes of pure braids (see Theorem 2.9). In other words, the set of link-homotopy classes of links is an orbit space of a certain group action. At this stage, we were greatly helped in defining the combinatorial problem explicitly by a suggestion of Levine. He conjectured a Markov-type theorem which turned out to be a straight-forward application of our classification theorem. The Levine moves are generators for the group action in our classification theorem (see Theorem 2.13).

Notice that in the classical Markov theorem (see [B]), one jumps between braid groups with different multiplicities. In contrast to that, we always use a fixed pure braid group, i.e., the Levine moves never increase nor decrease the multiplicity. This feature makes the combinatorial analysis of the Levine moves much easier than that of the classical Markov moves.

The combinatorial analysis of the Levine moves shows that the classification picture for links up to link-homotopy is analogous to that for maps between spaces. That is, for two links to be link-homotopic, one can inductively 
define certain "difference obstructions" which are cosets lying in certain free abelian groups. We show that these obstructions are inductively computable, thus providing an algorithm for determining whether two links (or link diagrams) are link-homotopic (see Theorem 3.1). A further consequence is that the link-homotopy classes of links form a recursively enumerable set.

This work was done in 1987 when the authors were in residence at the University of California at San Diego and participants in Freedman's topology seminar. We are grateful for the stimulating environment he provided us during that time. Lectures by Cochran $[\mathrm{C}]$ on Milnor's $\bar{\mu}$-invariants provided us with an understanding of the state of the art at the time as well as an appetite to learn more. Orr [O] lectured on the (still developing) obstruction theoretic picture for concordance.

Both these authors understood that the first nonvanishing invariants are additive. Cochran's construction of links, and his procedure for killing the $\bar{\mu}$ invariants, led us to the hope that one might be able to gain insight into the classification by looking at pictures. In the end, thanks to the algebra of the braid group, the necessary pictures turned out not to be so complicated.

The organization of the paper is as follows.

We will begin in $\S 1$ with generalities on links of 1-disks in the 3-disk, a notion we have decided to call string links. It turns out that the group of link-homotopy classes of string links is the same as the group of link-homotopy classes of pure braids with the same multiplicity. We were aided in our study of string links up to link-homotopy by use of Milnor's quotient group of the free group, which we will call the reduced free group. We can then represent the group of linkhomotopy classes of string links as the subgroup of the automorphism group of the reduced free group consisting of those automorphisms which satisfy two conditions similar to the conditions required for an automorphism of the free group to be induced by a pure braid. See Theorem 1.7. The proof of this theorem also makes the structure of the group of link-homotopy classes of string links quite clear.

Section 2 is devoted to the study of when the closures of two different string links (up to link-homotopy) give us the same link up to link-homotopy. Inside the group of link-homotopy classes of string links with a fixed multiplicity, we define, in addition to the usual relation of being conjugate, another relation among elements, which we call partial conjugacy. We show that the closures of two elements in the above group give the same link up to link-homotopy iff these two elements can be related via a sequence of elements in the same group such that any two adjacent elements in this sequence are either conjugate or partially conjugate. See Theorem 2.13 .

In $\S 3$, we carry out the combinatorial analysis of the equivalence relation generated by conjugacy and partial conjugacy. The output of this analysis is an algorithm which will decide whether two elements in the group of link-homotopy classes of string links are equivalent under this equivalence relation and thus accomplish our algorithmic classification of links up to link-homotopy. 


\section{STRING LINKS}

String links are a generalization of Artin's pure braids. One can think of a pure braid as a self-isotopy of $k$ points in the unit disk. A string link is a self-concordance of these $k$ points in the product of the unit disk and the unit interval.

Let $D$ be the unit disk in the plane and let $I=[0,1]$ be the unit interval. Choose $k$ points, $p_{1}, \ldots, p_{k}$, in the interior of $D$. Then, $\pi_{1}\left(D \backslash\left\{p_{1}, \ldots, p_{k}\right\}\right)$ is the free group $F(k)$ with $k$ generators. For definiteness, we choose $p_{1}, \ldots$, $p_{k}$ to lie in order on the $x$-axis and $e=(0,1)$ to be the base point. The loops $x_{i}$ shown in Figure 1.1 represent a preferred set of generators for $F(k)$. Note that the boundary of $D$ represents the product $x_{1} \cdots x_{k}$.

Let $I_{1}, \ldots, I_{k}$ be $k$ copies of the unit interval $I$. Denote by $\bigsqcup_{i=1}^{k} I_{i}$ the disjoint union of these intervals.

Definition 1.1. A string link is a smooth or piecewise linear proper imbedding

$$
\sigma: \coprod_{i=1}^{k} I_{i} \rightarrow D \times I
$$

such that $\left.\sigma\right|_{I_{i}}(0)=p_{i} \times 0$ and $\left.\sigma\right|_{I_{i}}(1)=p_{i} \times 1$. The image of $I_{i}$ is called the $i$ th string of the string link $\sigma$.

In Figure 1.2, we see a planar projection of a string link with two strings. Notice that each string of a string link inherits an orientation from the usual orientation of $[0,1]$.

Given a string link $\sigma$, we denote by

$$
Y=(D \times I) \backslash \sigma\left(\coprod_{i=1}^{k} I_{i}\right)
$$

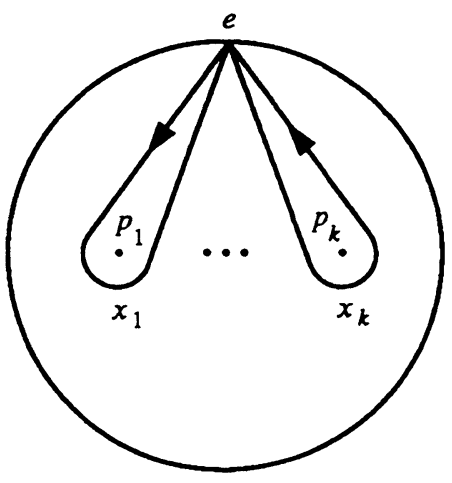

FIGURE 1.1 


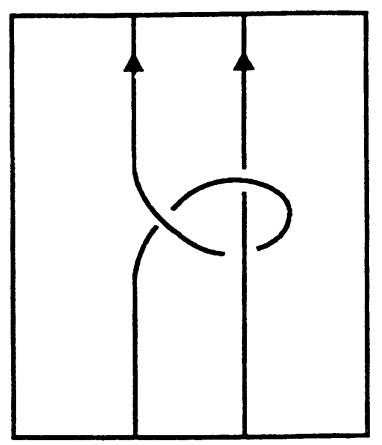

FIGURE 1.2

the complement of the strings, and by

$$
Y_{1}=\left(D \backslash\left\{p_{1}, \ldots, p_{k}\right\}\right) \times 1
$$

and

$$
Y_{0}=\left(D \backslash\left\{p_{1}, \ldots, p_{k}\right\}\right) \times 0
$$

the top and bottom boundaries of $Y$.

A theorem of Stallings [S] implies that the inclusion maps

$$
Y_{0} \rightarrow Y \leftarrow Y_{1}
$$

induce isomorphisms of the lower central series quotients of the fundamental groups

$$
\frac{\pi_{1}\left(Y_{0}\right)}{\left(\pi_{1}\left(Y_{0}\right)\right)_{n}} \cong \frac{\pi_{1}(Y)}{\left(\pi_{1}(Y)\right)_{n}} \cong \frac{\pi_{1}\left(Y_{1}\right)}{\left(\pi_{1}\left(Y_{1}\right)\right)_{n}}
$$

for all finite $n$. Here $\left\{G_{n} ; n=1,2, \ldots\right\}$ is the lower central series of a group $G$.

Recall that if $\sigma$ is a pure braid, the inclusions $Y_{0} \rightarrow Y \leftarrow Y_{1}$ induce isomorphisms

$$
\pi_{1}\left(Y_{0}\right) \cong \pi_{1}(Y) \cong \pi_{1}\left(Y_{1}\right)
$$

and after identifying $\pi_{1}\left(Y_{i}\right)$ with $F(k)$, the resulting automorphism $\phi_{\sigma}$ of $F(k)$ will satisfy

(1) $\phi_{\sigma}\left(x_{i}\right)$ is conjugate to $x_{i}$ for $i=1, \ldots, k$; and

(2) $\phi_{\sigma}\left(x_{1} \cdots x_{k}\right)=x_{1} \cdots x_{k}$.

Artin's representation theorem establishes an isomorphism between the pure braid group with multiplicity $k$ and the subgroup of the automorphism group of $F(k)$ (acting on the right) consisting of those automorphisms satisfying (1) and (2). See [B].

For a string link $\sigma$, the group $\pi_{1}(Y)$ is in general larger than the free group. However, the isomorphisms in $(*)$ show how to eliminate this difficulty to some extent. One would hope to establish Artin-type theorems for string links using these quotients. What is lacking is a geometric description of the relation this 
imposes on string links. We will obtain an Artin-type theorem by considering another nilpotent quotient, introduced by Milnor [M1].

Definition 1.2. Let $G$ be a group normally generated by $x_{1}, \ldots, x_{k}$. We denote by $R G$ the quotient group obtained by adding relations which say each $x_{i}$ commutes with all of its conjugates. We call $R F(k)$ the reduced free group.

Remark. Let $N_{i}$ be the normal subgroup of $G$ generated by $x_{i}$ and let $\left[N_{i}\right]$ denote the commutator subgroup of $N_{i}$. Then $N=\left[N_{1}\right] \cdots\left[N_{k}\right]$ is also normal and $R G=G / N$. Thus $R G$ depends only on the conjugacy classes of $x_{i}$, $i=1, \ldots, k$. Moreover, the normal subgroup $\bar{N}_{i}$ of $R G$ generated by the image $\bar{x}_{i}$ of $x_{i}$ is abelian.

Lemma 1.3. $R G$ is nilpotent of class $\leq k$.

Proof. Let $\bar{x}_{i}$ denote the image of $x_{i}$ in $R G$. Let $N_{i}$ and $\bar{N}_{i}$ denote the normal subgroups generated by $x_{i}$ and $\bar{x}_{i}$, respectively. As $G / N_{i}$ is normally generated by the images of $x_{j}, j \neq i$, we may inductively assume that $R\left(G / N_{i}\right)$ is nilpotent of class $\leq k-1$. It is easily checked that $R G / \bar{N}_{i}=R\left(G / N_{i}\right)$. Thus if $g \in(R G)_{k}, g$ maps trivially into $R G / \bar{N}_{i}$, i.e., $g$ lies in the abelian group $\bar{N}_{i}$. But $R G$ is generated by these abelian subgroups, hence $g$ lies in the center of $R G$. Hence $(R G)_{k}$ is central and $(R G)_{k+1}=1$.

Corollary 1.4. The inclusions $Y_{0} \rightarrow Y \leftarrow Y_{1}$ induce isomorphisms

$$
R \pi_{1}\left(Y_{0}\right) \cong\left(\pi_{1}(Y) \cong R \pi_{1}\left(Y_{1}\right)\right. \text {. }
$$

Remark. If $x_{i}^{0}$ 's are the preferred generators of $\pi_{1}\left(Y_{0}\right)$ and $x_{i}^{1}$ 's those of $\pi_{1}\left(Y_{1}\right)$, then the images of $x_{i}^{0}$ and $x_{i}^{1}$ are conjugate in $\pi_{1}(Y)$ and normally generate $\pi_{1}(Y)$. Hence the notation $R \pi_{1}(Y)$ is unambiguously defined.

Proof of Corollary 1.4. Using Lemma 1.3, one easily checks that $R(G)=$ $R\left(G / G_{n}\right)$ for $n \geq k$. Hence the isomorphisms in $(*)$ for $n \geq k$ imply that of Corollary 1.4 .

Let $\mathscr{A}(k)$ denote the group consisting of all automorphisms $\phi$ of $R F(k)$ satisfying

(1) $\phi\left(x_{i}\right)$ is conjugate to $x_{i}$ for $i=1, \ldots, k$; and

(2) $\phi\left(x_{1} \cdots x_{k}\right)=x_{1} \cdots x_{k}$.

Identifying $R \pi_{1}\left(Y_{i}\right)$ with $R F(k)$, Corollary 1.4 associates to each string link $\sigma$ an automorphism $\phi_{\sigma}$ of $R F(k)$ which satisfies (1) and (2), i.e., $\phi_{\sigma} \in \mathscr{A}(k)$.

Definition 1.5. We say that two string links $\sigma$ and $\sigma^{\prime}$ are link-homotopic, if there is a homotopy of the strings in $D \times I$, fixing the endpoints and deforming $\sigma$ to $\sigma^{\prime}$, such that the images of different strings remain disjoint during the deformation.

Remark. A general link-homotopy can always be approximated by one which is given by a sequence of ambiant isotopies of $D \times I$, fixing the boundary, and 


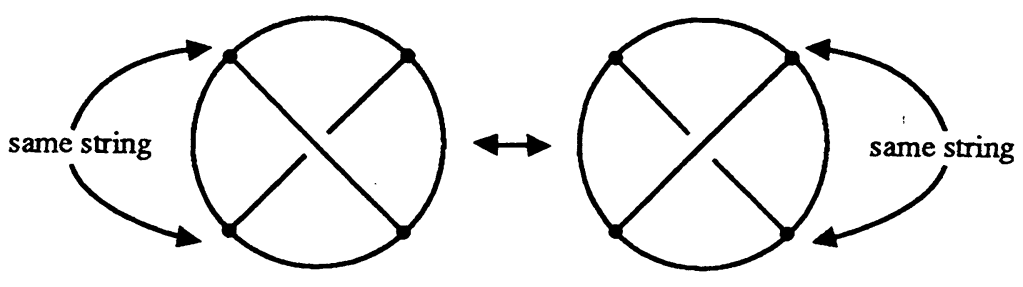

FIGURE 1.3

"crossing changes." A crossing change is a homotopy of a single string of a string link supported in a ball whose intersection with the string link consists of two segments of that string which cross during the deformation. See Figure 1.3.

We will denote by $\mathscr{H}(k)$ the link-homotopy classes of string links with $k$ strings.

Lemma 1.6. If $\sigma$ and $\sigma^{\prime}$ are link-homotopic string links, then $\phi_{\sigma}=\phi_{\sigma^{\prime}}$.

Proof. Let $Y$ and $Y^{\prime}$ denote the complements of the string links $\sigma$ and $\sigma^{\prime}$ respectively, and let $Z \subset(D \times I) \times I$ denote the complement of a homotopy from $\sigma$ to $\sigma^{\prime}$, which we take to be either an isotopy or a crossing change. We will show that the inclusions

$$
Y \rightarrow Z \leftarrow Y^{\prime}
$$

induce isomorphisms on $R \pi_{1}$. Assuming this, we obtain a commutative diagram

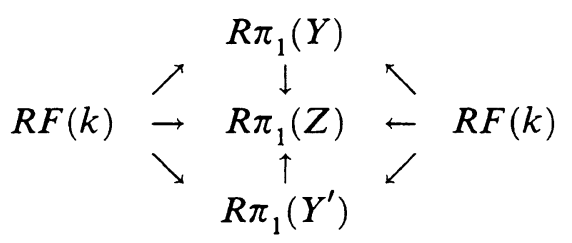

of isomorphisms, showing that $\phi_{\sigma}=\phi_{\sigma^{\prime}}$.

For an isotopy, the inclusions already induce isomorphisms on $\pi_{1}$ and hence on $R \pi_{1}$. For a crossing change, assume the crossing occurs at time $t=\frac{1}{2}$ and let $Y_{1 / 2}$ be the complement of the image of the strings at time $t=\frac{1}{2}$. Note that up to homotopy type, $Y_{1 / 2}$ can be obtained from $Y$ by removal of a segment $s$ joining the strings to be crossed. See Figure 1.4. Dually, $Y$ is obtained from $Y_{1 / 2}$ by adding a 2-cell transversal to $s$. Similarly, $Y^{\prime}$ is obtained from $Y_{1 / 2}$ by adding a 2-cell and $Z$ can be obtained from $Y_{1 / 2}$ by adding both these cells.

Let $B^{3}$ denote a ball containing the crossing change. Then $B^{3} \cap Y_{1 / 2}$ deformation retracts to $S^{2}$ with four points removed. The fundamental group of this punctured sphere has generators $a, b, c, d$ subject to the relation $a b c d=1$. See Figure 1.5.

Note that $B^{3} \cap Y$ is obtained from $B^{3} \cap Y_{1 / 2}$ by attaching a 2-cell along the curve $a c$ and $\left(B^{3} \times I\right) \cap Z$ is obtained from $B^{3} \cap Y_{1 / 2}$ by attaching 2-cells along 

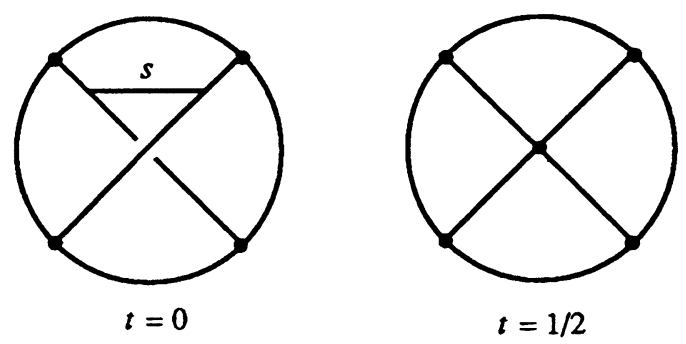

FIGURE 1.4

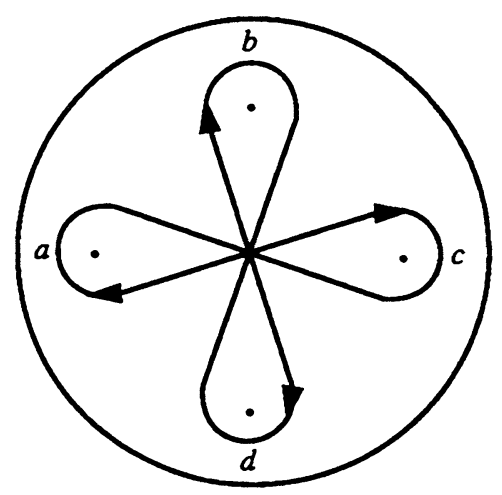

FIGURE 1.5

the curves $a c$ and $b d$. Hence

$$
\pi_{1}(Z)=\frac{\pi_{1}\left(Y_{1 / 2}\right)}{\langle a c, b d\rangle}=\frac{\pi_{1}(Y)}{\langle b d\rangle},
$$

and

$$
R \pi_{1}(Z)=\frac{R \pi_{1}(Y)}{\langle b d\rangle} .
$$

(Here $\left\langle g_{1}, g_{2}, \ldots, g_{r}\right\rangle$ is the normal subgroup of a group $G$ normally generated by the elements $g_{1}, g_{2}, \ldots, g_{r}$ of $G$.) But in $R \pi_{1}(Y), b$ and $c$ are conjugates of one of the generators and hence commute. So the relation $a b c d=1$ can be written as $a c b d=1$. Thus the relation $b d=1$ already holds in $R \pi_{1}(Y)$ since the relation $a c=1$ holds. Therefore,

$$
R \pi_{1}(Z)=\frac{R \pi_{1}(Y)}{\langle b d\rangle}=R \pi_{1}(Y) .
$$

Similarly $R \pi_{1}\left(Y^{\prime}\right) \rightarrow R \pi_{1}(Z)$ is an isomorphism.

Lemma 1.6 shows that the correspondence $\sigma \mapsto \phi_{\sigma}$ induces a map $\mathscr{H}(k)$ $\rightarrow \mathscr{A}(k)$. 
The product of two string links $\sigma$ and $\sigma^{\prime}$, denoted by $\sigma \sigma^{\prime}$, is given by stacking $\sigma^{\prime}$ on the top of $\sigma$ and reparametrizing. Specifically,

$$
\left.\left(\sigma \sigma^{\prime}\right)\right|_{I_{i}}= \begin{cases}\rho\left(\left.\sigma\right|_{I_{i}}(2 t)\right), & \text { for } 0 \leq t \leq \frac{1}{2}, \\ \rho^{\prime}\left(\left.\sigma^{\prime}\right|_{I_{i}}(2 t-1)\right), & \text { for } \frac{1}{2} \leq t \leq 1,\end{cases}
$$

where $\rho, \rho^{\prime}: D \times I \rightarrow D \times I$ are given by

$$
\rho(z, t)=\left(z, \frac{1}{2} t\right)
$$

and

$$
\rho^{\prime}(z, t)=\left(z, \frac{1}{2} t+\frac{1}{2}\right) .
$$

With this product, $\mathscr{H}(k)$ inherits an associative multiplication with unit given by the trivial string link 1 , where

$$
\left.\mathbf{1}\right|_{I_{i}}(t)=\left(p_{i}, t\right) \text {. }
$$

We define the inverse $\sigma^{-1}$ of a string link $\sigma$ by

$$
\left.\sigma^{-1}\right|_{I_{i}}(t)=r\left(\left.\sigma\right|_{I_{i}}(1-t)\right)
$$

where $r: D \times I \rightarrow D \times I$ is given by

$$
r(z, t)=(z, 1-t) .
$$

We shall see that the link-homotopy class of $\sigma^{-1}$, denoted by $\left[\sigma^{-1}\right]$, is the right and left inverse for $[\sigma]$ in $\mathscr{H}(k)$ (see the proof of Lemma 1.8 below). Hence $\mathscr{H}(k)$ is a group. Moreover, $\mathscr{H}(k) \rightarrow \mathscr{A}(k)$ is a homomorphism of groups $(\mathscr{A}(k)$ is acting on the right of $R F(k))$.

The main result of this section is the following.

Theorem 1.7. The map

$$
\begin{aligned}
\mathscr{H}(k) & \rightarrow \mathscr{A}(k) \\
\sigma & \mapsto \phi_{\sigma}
\end{aligned}
$$

is an isomorphism.

Let us first prove the following

Lemma 1.8. There is a split short exact sequence of groups

$$
1 \rightarrow R F(k-1) \rightarrow \mathscr{H}(k) \rightarrow \mathscr{H}(k-1) \rightarrow 1 .
$$

Proof. The map $\mathscr{H}(k) \rightarrow \mathscr{H}(k-1)$ is defined by omitting the $k$ th string. A splitting is given by adding a trivial $k$ th string.

The map $R F(k-1) \rightarrow \mathscr{H}(k)$ is defined as follows. Let $\lambda \in F(k-1)$ be represented by a path $\gamma$ in $D \backslash\left\{p_{1}, \ldots, p_{k-1}\right\}$ based at $p_{k}$. Then a string link $\sigma_{\lambda}$ is defined by

$$
\left.\sigma_{\lambda}\right|_{I_{i}}(t)= \begin{cases}\left.1\right|_{I_{i}}(t), & \text { for } i<k \\ (\gamma(t), t), & \text { for } i=k\end{cases}
$$




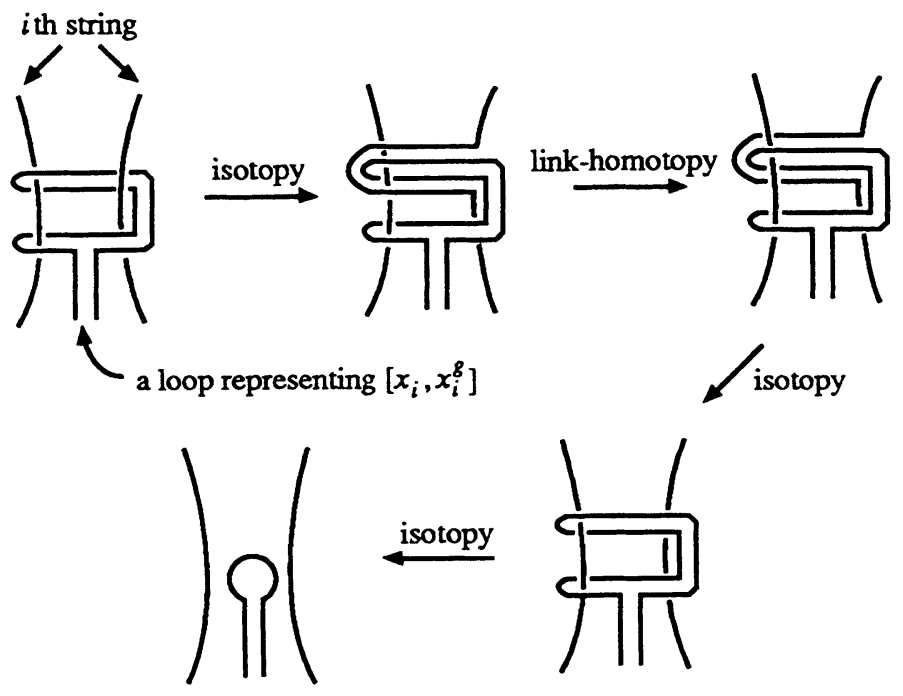

FIGURE 1.6

Note that $\sigma_{\lambda}$ is a pure braid whose first $k-1$ strings are trivial, and whose isotopy class is well defined by $\lambda$. This defines a homomorphism whose image is easily seen to be onto the kernel of $\mathscr{H}(k) \rightarrow \mathscr{H}(k-1)$.

(At this point, it follows that $\mathscr{H}(k)$ is a group since it is a split extension of one group by another. In fact, from what we have just seen it follows that it is a quotient of the pure braid group. For a pure braid $\sigma,\left[\sigma^{-1}\right]=[\sigma]^{-1}$ as claimed.)

Now if $\lambda$ is in the kernel of $F(k-1) \rightarrow R F(k-1)$, then $\lambda$ is a product of conjugates of the elements $\left[x_{i}, x_{i}^{g}\right], g \in F(k-1)$. A geometric argument of Milnor [M1] shows that $\sigma_{\lambda}$ is link-homotopic to 1 . This geometric argument is displayed in Figure 1.6. So the map $F(k-1) \rightarrow \mathscr{H}(k)$ factors to give a map $R F(k-1) \rightarrow \mathscr{H}(k)$.

To see that this map is injective, let $\sigma$ be any string link with $k$ strings and $\bar{\sigma}$ the string link obtained by omitting the $k$ th string. Let $\bar{Y}$ denote its complement. Then the $k$ th string represents an element in $\pi_{1}(\bar{Y})$. (Specifically, connect the ends of the $k$ th string to the "base point" $e \times I$ along the straight line segments (see Figure 2.1) $a_{k} \times 0, a_{k} \times 1$, between $e \times 0, e \times 1$ and $p_{k} \times 0$, $p_{k} \times 1$ respectively.) Using the isomorphism

$$
R F(k-1)=R \pi_{1}\left(\bar{Y}_{1}\right) \cong R \pi_{1}(\bar{Y})
$$

we obtain an element $\lambda_{\sigma} \in R F(k-1)$. Clearly $\lambda \mapsto\left[\sigma_{\lambda}\right] \mapsto \lambda_{\sigma}$ is the identity map of $R F(k-1)$, hence $R F(k-1) \rightarrow \mathscr{H}(k)$ is injective.

Remark. The correspondence $\sigma \mapsto \lambda_{\sigma}$ induces a projection $\mathscr{H}(k) \rightarrow R F(k-1)$ corresponding to the splitting $\mathscr{H}(k-1) \rightarrow \mathscr{H}(k)$ and is not a homomorphism. 
A modified version of the above proof establishes the split short exact sequence

$$
1 \rightarrow F(k-1) \rightarrow P B(k) \rightarrow P B(k-1) \rightarrow 1
$$

where $P B(k)$ is the pure braid group on $k$ strings. Consequently,

$$
P B(k)=(\cdots(F(1) \ltimes F(2)) \ltimes \cdots) \ltimes F(k-1)
$$

and

$$
\mathscr{H}(k)=(\cdots(R F(1) \ltimes R F(2)) \ltimes \cdots) \ltimes R F(k-1)
$$

with the map $P B(k) \rightarrow \mathscr{H}(k)$ being the obvious projection. Since the projections $F(i) \rightarrow R F(i), i=1, \ldots, k-1$, are onto, the projection $P B(k) \rightarrow \mathscr{H}(k)$ is also onto. A consequence of this is that any string link is link-homotopic to a pure braid.

In his 1946 paper, "The theory of braids" [A], Artin asked the question whether the notion of isotopy and homotopy (link-homotopy here) of braids are different. This was established only in 1973, when Goldsmith [G1] essentially established the above semidirect product decomposition for $\mathscr{H}(k)$, by introducing relations in $P B(k)$ corresponding to the projections $F(i) \rightarrow R F(i)$.

Before we can prove Theorem 1.7, we need to know a bit more about the structure of the group $R F(k)$. In [M], Milnor constructed a polynomial ring $P(k)$ in noncommutative variables $X_{1}, \ldots, X_{k}$ such that monomials with two or more occurrences of some variable are set to zero. Milnor showed that

$$
\left\{1, X_{i_{1}} X_{i_{2}} \cdots X_{i_{r}} ; 1 \leq i_{1}, i_{2}, \ldots, i_{r} \leq k, i_{s} \neq i_{t} \text { if } s \neq t\right\}
$$

forms a free basis for the abelian group $P(k)$. We then have an injective expansion $R F(k) \rightarrow P(k)$ given by sending $x_{i}$ to $1+X_{i}$. This expansion is actually a reduction of the well-known Magnus expansion for the free group $F(k)$ [MKS]. Notice the expansion of $x_{i}^{-1}$ is $1-X_{i}$.

Let $\lambda \in R F(k), \lambda \neq 1$, then $\delta(\lambda) \in P(k)$ is defined to be the nonvanishing homogeneous component of the lowest positive degree in the expansion of $\lambda$. We define $\delta(1)=0$.

Lemma 1.9. Suppose $\lambda \in R F(k)$, then

(1) $\delta\left(\left[\lambda, x_{i}\right]\right)=\delta(\lambda) X_{i}-X_{i} \delta(\lambda)$

(2) $\delta\left(\lambda^{-1}\right)=-\delta(\lambda)$;

(3) $\delta\left(\lambda^{x_{i}}\right)=\delta(\lambda)$;

(4) if $\operatorname{deg} \delta(\lambda)=\operatorname{deg} \delta(\mu)$, then $\delta(\lambda \mu)=\delta(\lambda)+\delta(\mu)$; if $\operatorname{deg} \delta(\lambda)<\operatorname{deg} \delta(\mu)$, then $\delta(\lambda \mu)=\delta(\lambda)$.

Proof. An easy computation.

Remark. It is the additivity property (4) that is responsible in part for the additivity of the first nonvanishing $\bar{\mu}$-invariants.

Lemma 1.10. The centralizer of $x_{i}$ in $R F(k)$ is $N_{i}$, the normal subgroup normally generated by $x_{i}$. 
Proof. $N_{i}$ is abelian and so centralizes $x_{i}$. Consider the split exact sequence

$$
1 \rightarrow N_{i} \rightarrow R F(k) \rightarrow R F(k-1) \rightarrow 1 .
$$

Here $R F(k-1)=R F(k) / N_{i}$. It suffices to see that no element of $R F(k-1) \subset$ $R F(k)$ centralizes $x_{i}$. Let $\lambda \in R F(k-1)$ with $\lambda \neq 1$. Then $\delta(\lambda) \neq 0$ and the monomials in $\delta(\lambda)$ contain no $X_{i}$. So $\delta\left(\left[\lambda, x_{i}\right]\right) \neq 0$ according to Lemma 1.9. Consequently, $\left[\lambda, x_{i}\right] \neq 1$.

Proof of Theorem 1.7. Suppose $\phi \in \mathscr{A}(k)$, then $\phi\left(x_{i}\right)=x_{i}^{\lambda_{i}}$ for some $\lambda_{i} \in$ $R F(k), i=1, \ldots, k$. Clearly, $\lambda_{i}$ is well defined up to multiplication on the right by centralizers of $x_{i}$. By Lemma 1.10, it follows that the image of $\lambda_{i}$, denoted by $\bar{\lambda}_{i}$, in $R F(k-1)=R F(k) / N_{i}$ is well defined. It should be remarked that $\bar{\lambda}_{i} \in R F(k-1) \subset R F(k)$ is a canonical choice satisfying $\phi\left(x_{i}\right)=x_{i}^{\bar{\lambda}_{i}}$ and $\left\{\bar{\lambda}_{1}, \ldots, \bar{\lambda}_{k}\right\}$ is determined uniquely by $\phi$.

We define $\Lambda: \mathscr{A}(k) \rightarrow R F(k-1)$ as follows. If $\phi \in \mathscr{A}(k)$, then $\Lambda(\phi)=\bar{\lambda}_{k}$ where $\bar{\lambda}_{k}$ is the unique element in $R F(k-1)=R F(k) / N_{k}$ such that $\phi\left(x_{k}\right)=$ $x_{k}^{\bar{\lambda}_{k}}$.

The quotient map $R F(k) \rightarrow R F(k-1)=R F(k) / N_{k},-\lambda \mapsto \bar{\lambda}$ induces an epimorphism $\mathscr{A}(k) \rightarrow \mathscr{A}(k-1)$. The image of $\phi \in \mathscr{A}(k)$ will be denoted by $\bar{\phi}$. We have

$$
\overline{\phi(\lambda)}=\bar{\phi}(\bar{\lambda}), \quad \phi \in \mathscr{A}(k), \lambda \in R F(k) .
$$

Remember that we have defined the action of $\mathscr{A}(k)$ on $R F(k)$ to be a right action. So if $\phi, \phi^{\prime} \in \mathscr{A}(k)$, we have

$$
\left(\left(x_{k}\right) \phi\right) \phi^{\prime}=\left(x_{k}^{\lambda_{k}}\right) \phi^{\prime}=x_{k}^{\left(\lambda_{k}\right) \phi^{\prime} \cdot \lambda_{k}^{\prime}} .
$$

Thus

$$
\begin{aligned}
\Lambda\left(\phi \phi^{\prime}\right) & =\overline{\left(\lambda_{k}\right) \phi^{\prime} \cdot \lambda_{k}^{\prime}}=\overline{\left(\lambda_{k}\right) \phi^{\prime}} \cdot \bar{\lambda}_{k}^{\prime} \\
& =\left(\bar{\lambda}_{k}\right) \phi^{\prime} \cdot \bar{\lambda}_{k}^{\prime}=(\Lambda(\phi)) \bar{\phi}^{\prime} \cdot \Lambda\left(\phi^{\prime}\right) .
\end{aligned}
$$

From this, it is easy to see that the map $\Lambda$ restricted on the kernel $K$ of $\mathscr{A}(k) \rightarrow \mathscr{A}(k-1)$ is a homomorphism.

We have the following diagram

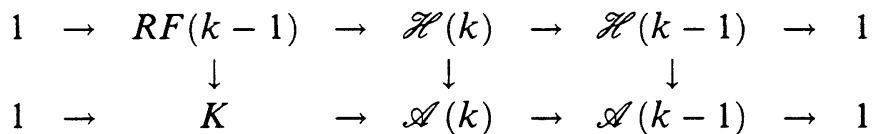

$$
\begin{aligned}
& \Lambda \downarrow \\
& R F(k-1)
\end{aligned}
$$

with exact rows. The composition $R F(k-1) \rightarrow R F(k-1)$ is the identity, since for any string link $\sigma$, the $k$ th string considered as a loop in the complement of the first $k-1$ strings represents a word in $R F(k-1)$ which is exactly $\Lambda\left(\phi_{\sigma}\right)$.

Since inductively $\mathscr{H}(k-1) \rightarrow \mathscr{A}(k-1)$ is an isomorphism, it remains to show that $\Lambda$ is injective on $K$. So let $\phi \in K, \phi\left(x_{i}\right)=x_{i}^{\bar{\lambda}_{i}}, i=1, \ldots, k$. 
As $\bar{\lambda}_{k}=1$, we have $\phi\left(x_{k}\right)=x_{k}$. We must show $\phi\left(x_{i}\right)=x_{i}$ for $i<k$. Or equivalently $\bar{\lambda}_{i}=1$ for $i<k$. Now we have

$$
\phi\left(x_{1} \cdots x_{k}\right)=x_{1} \cdots x_{k} \text {. }
$$

So we obtain

$$
\left[\lambda_{1}, x_{1}\right] x_{1} \cdots\left[\lambda_{k-1}, x_{k-1}\right] x_{k-1}=x_{1} \cdots x_{k-1}
$$

i.e.,

$$
\alpha=\left[\lambda_{1}, x_{1}\right]\left[\lambda_{2}, x_{2}\right]^{x_{1}} \cdots\left[\lambda_{k-1}, x_{k-1}\right]^{x_{1} \cdots x_{k-2}}=1 \text {. }
$$

We will consider $\delta\left(\bar{\lambda}_{i}\right), i=1, \ldots, k-1$. Notice $\operatorname{deg} \delta\left(\bar{\lambda}_{i}\right) \leq k-1$ and the monomials in $\delta\left(\bar{\lambda}_{i}\right)$ contain no $X_{i}$. On the other hand, since $\phi \in K$, each monomial of $\delta\left(\bar{\lambda}_{i}\right)$ contains $X_{k}$ (if $\delta\left(\bar{\lambda}_{i}\right) \neq 0$ ). Assume for the purpose of reaching a contradiction that the set $J=\left\{i ; \bar{\lambda}_{i} \neq 1\right\}$ is nonempty. Let $I$ be the subset of $J$ consisting of those indices $i$ with $\operatorname{deg} \delta\left(\bar{\lambda}_{i}\right)$ minimal. Then, since $\delta(\alpha)=0$, using Lemma 1.9 , we get

$$
\sum_{i \in I}\left(\delta\left(\bar{\lambda}_{i}\right) X_{i}-X_{i} \delta\left(\bar{\lambda}_{i}\right)\right)=0
$$

Suppose $i_{1} \in I$ and $M_{i_{1}}$ is a monomial in $\delta\left(\bar{\lambda}_{i_{1}}\right)$. In the above expression, the monomial $M_{i_{1}} X_{i_{1}}$ can only be canceled using the monomials in $X_{i} \delta\left(\bar{\lambda}_{i}\right)$, $i \neq i_{1}$. So there must be a monomial $M_{i_{2}}$ in $\delta\left(\lambda_{i_{2}}\right), i_{2} \in I \backslash\left\{i_{1}\right\}$, with $M_{i_{1}} X_{i_{1}}=$ $X_{i_{2}} M_{i_{2}}$. In order to cancel $M_{i_{2}} X_{i_{2}}$, there must be a monomial $M_{i_{3}}$ in $\delta\left(\lambda_{i_{3}}\right)$, $i_{3} \in I \backslash\left\{i_{2}\right\}$, with $M_{i_{2}} X_{i_{2}}=X_{i_{3}} M_{i_{3}}$. We claim $i_{3} \neq i_{1}$. This is because the last variable in $M_{i_{2}}$ is $X_{i_{1}}$ which cannot be the first variable as $M_{i_{2}}$ contains $X_{k}$ and there are no repeated indices. Note that the last two variables of $M_{i_{3}}$ are $X_{i_{1}}$ and $X_{i_{2}}$ so that there is an $i_{4}$ in $I \backslash\left\{i_{1}, i_{2}, i_{3}\right\}$ by the same reason. Continuing in this way, we eventually find that the cardinality of $I$ is greater than $k-1$, a contradiction. This contradiction shows that we must have had

$$
\bar{\lambda}_{1}=\cdots=\bar{\lambda}_{k-1}=1
$$

and proves Theorem 1.7.

Corollary 1.11. We have

$$
\mathscr{A}(k)=\mathscr{A}(k-1) \ltimes R F(k-1)
$$

where the action of $\mathscr{A}(k-1)$ on $R F(k-1)$ is the natural right action.

Proof. The splitting $\mathscr{A}(k-1) \rightarrow \mathscr{A}(k)$ is defined by

$$
\phi\left(x_{i}\right)= \begin{cases}\bar{\phi}\left(x_{i}\right) & \text { for } i<k, \\ x_{k}, & \text { for } i=k,\end{cases}
$$

where $\bar{\phi}\left(x_{i}\right) \in R F(k-1) \subset R F(k)$. This clearly defines a map $F(k) \rightarrow$ $R F(k)$ which descends to a map $R F(k) \rightarrow R F(k)$ inducing the identity after abelianization. Since $R F(k)$ is nilpotent, it must be an isomorphism. 
Suppose $\bar{\phi} \in \mathscr{A}(k-1), \varphi \in K$. Note that

$$
\Lambda\left(\phi^{-1}\right)=\Lambda(\phi)=1 .
$$

Hence we have

$$
\begin{aligned}
\Lambda\left(\phi^{-1} \varphi \phi\right) & =\left(\Lambda\left(\phi^{-1}\right)\right) \overline{\varphi \phi} \cdot \Lambda(\varphi \phi)=\Lambda(\varphi \phi) \\
& =(\Lambda(\varphi)) \bar{\phi} \cdot \Lambda(\phi)=(\Lambda(\varphi)) \bar{\phi}
\end{aligned}
$$

showing that the action is the natural right action.

Proposition 1.12. $\mathscr{A}(k)$ is torsion free and nilpotent of class $k-1$. Moreover, we have

$$
\mathscr{A}(k)_{i}=\mathscr{A}(k-1)_{i} \ltimes R F(k-1)_{i} .
$$

Proof. We know $R F(k-1)$ is torsion free and nilpotent of class $k-1$. Hence $\mathscr{A}(k)$ is torsion free. As the action of $\mathscr{A}(k-1)$ on the abelianization of $R F(k-1)$ is trivial, it acts trivially on $R F(k-1)_{i} / R F(k-1)_{i+1}$ for all $i$. It follows that

$$
\mathscr{A}(k)_{i}=\mathscr{A}(k-1)_{i} \ltimes R F(k-1)_{i} .
$$

Hence $\mathscr{A}(k)_{k-1}=R F(k-1)_{k-1} \neq 1$ and $\mathscr{A}(k)_{k}=R F(k-1)_{k}=1$, so $\mathscr{A}(k)$ is nilpotent of class $k-1$.

Let us quote a result of Levine here which gives a basis for the torsion free nilpotent group $R F(k)$. In particular, it shows that the word problem in $R F(k)$ and hence in $\mathscr{A}(k)$ is solvable.

Proposition 1.13. Given a Hall basis of basic commutators for the free group $F(k)$, let $C_{1}, \ldots, C_{n}$ be the ordered subset of basic commutators in $\left\{x_{1}, \ldots, x_{k}\right\}$ without repeated appearances of the variables. Then each element of $R F(k)$ can be written uniquely as a product $C_{1}^{e_{1}} \ldots C_{n}^{e_{n}}$ where $e_{1}, \ldots, e_{n}$ are integers.

Proof. See [Le2].

Next we give a geometric interpretation of the lower central series of the group $\mathscr{A}(k)$. This also gives an alternative proof of the nilpotency of $\mathscr{H}(k)$.

Definition 1.14. A string link $\sigma$ is called $r$-trivial if after omitting any set of $r$ strings from $\sigma$, the remaining strings form a link-homotopically trivial string link.

We denote by $\{\mathscr{H}(k)\}_{r}$ the normal subgroup of $\mathscr{H}(k)$ consisting of all $r$ trivial string links.

Proposition 1.15. We have

$$
\{\mathscr{H}(k)\}_{r}=\mathscr{H}(k)_{k-r} .
$$

Proof. Let $\sigma \in \mathscr{H}(k)_{k-r}$. After omitting $r$ strings from $\sigma$, it maps to an element in $\mathscr{H}(k-r)_{k-r}$ which inductively equals $\{\mathscr{H}(k-r)\}_{0}=1$. Hence $\mathscr{H}(k)_{k-r} \subset\{\mathscr{H}(k)\}_{r}$. 
For $\sigma \in\{\mathscr{H}(k)\}_{r}$, let $\sigma=\theta g$ with $\theta \in \mathscr{H}(k-1)$ and $g \in R F(k-1)$ corresponding to the semidirect decomposition $\mathscr{H}(k)=\mathscr{H}(k-1) \ltimes R F(k-1)$ where $R F(k-1)=R F(k) / N_{k}$. Omitting $r$ strings other than the $k$ th one gives a map to $\mathscr{H}(k-r)=\mathscr{H}(k-r-1) \ltimes R F(k-r-1)$ respecting the decomposition. Since $\sigma$ maps to 1 , it follows that $g$ maps to 1 . Since every basic commutator in the Levine normal form of $g$ of weight less than $k-r$ will be detected by some such map, it follows that there are no such commutators and hence $g \in R F(k-1)_{k-r} \subset \mathscr{H}(k)_{k-r}$.

Omitting $r$ strings including the $k$ th one from $\sigma$ amounts to omitting $r-1$ strings from $\theta$. Hence

$$
\theta \in\{\mathscr{H}(k-1)\}_{r-1}=\mathscr{H}(k-1)_{k-r} \subset \mathscr{H}(k)_{k-r} .
$$

Thus $\sigma=\theta g \in \mathscr{H}(k)_{k-r}$, i.e., $\{\mathscr{H}(k)\}_{r} \subset \mathscr{H}(k)_{k-r}$.

\section{ClASSIFYING LINKS UP TO LINK-HOMOTOPY}

A link in $S^{3}$ is a smooth or piecewise linear imbedding of a disjoint union of ordered, oriented circles $\coprod_{i=1}^{k} S_{i}^{1}$ into $S^{3}$. Let

$$
L: \coprod_{i=1}^{k} S_{i}^{1} \rightarrow S^{3}
$$

be an ordered, oriented link. We will also denote the image of $L$ by $L$ and will call $L_{i}=L\left(S_{i}^{1}\right)$ the $i$ th component of $L$.

Given a string link $\sigma$, we can close it up to get an ordered, oriented link $\hat{\sigma}$ in $S^{3}$. This process can be reversed, though not canonically, and the study of the resulting indeterminancy is the key to our classification.

Let $\pi$ denote the projection $D \times I \rightarrow D$. If we identify points on the boundary of $D \times I$ with their images under $\pi$, the resulting quotient space is homeomorphic to $S^{3}$. Moreover, after identifying $I / \partial I$ with $S^{1}$, the string link $\sigma$ gives rise to a link

$$
\hat{\sigma}: \coprod_{i=1}^{k} S_{i}^{1} \rightarrow S^{3}
$$

which inherits an ordering and orientation from $\sigma$. This link $\hat{\sigma}$ is called the closure of the string link $\sigma$.

Suppose the oriented unit disk $D$ is imbedded in $S^{3}$ such that its intersection with the $i$ th component of the link $L$ is $p_{i}, i=1, \ldots, k$, and all intersection numbers are +1 . Then we call the image of $D$ a $d$-base for $L$ and still denote it by $D$.

Definition 2.1. A $d$-based link is a pair $(L, D)$ where $L$ is a link and $D$ is a $d$-base of $L$.

For the closure $\hat{\sigma}$ of a string link $\sigma$, there is a canonical choice of a $d$-base $D=D \times 0=D \times 1$. By the $d$-based link $(\hat{\sigma}, D)$, we will always mean the link $\hat{\sigma}$ with its canonical $d$-base $D$. 
One can easily construct for any link $L$ a $d$-base. Once this has been done, one can easily convert a link to a string link. Thus we have

Lemma 2.2. For any link $L$, there is a string link $\sigma$ such that $\hat{\sigma}$ is isotopic to $L$.

We define two $d$-based links $(L, D)$ and $\left(L^{\prime}, D^{\prime}\right)$ to be (ambient) isotopic if there is an isotopy of $S^{3}$ which deforms $L \cup D$ to $L^{\prime} \cup D^{\prime}$ preserving the ordering and orientation of the components.

Lemma 2.3. The correspondence $\sigma \mapsto(\hat{\sigma}, D)$ induces a bijection between isotopy classes of string links and isotopy classes of $d$-based links.

Let us recall the definition of two links being link-homotopic as introduced by Milnor [M1] (his terminology was simply "homotopic"). See also [Le2]. Two links are link-homotopic (preserving the ordering and orientation of the components) if one can be deformed to the other through a sequence of ambient isotopies of $S^{3}$ (preserving the ordering and orientation of the components) and "crossing changes." As before, a crossing change is a homotopy of a single component of a link supported in a ball whose intersection with the link consists of two segments of that component which cross during the defomation.

We can also define a link-homotopy between two $d$-based links $(L, D)$ and $\left(L^{\prime}, D^{\prime}\right)$. It will be a link-homotopy from $L$ to $L^{\prime}$ which deforms $D$ to $D^{\prime}$ isotopically and no ball supporting a crossing change has intersection with the image of $D$ at the time that crossing change is being accomplished.

Corollary 2.4. The correspondence $\sigma \mapsto(\hat{\sigma}, D)$ induces a bijection between linkhomotopy classes of string links and link-homotopy classes of $d$-based links.

Figure 2.1 shows a system of arcs $\left\{a_{1}, \ldots, a_{k}\right\}$ in $D$ connecting the base point $e$ of $D$ to the points $\left\{p_{1}, \ldots, p_{k}\right\}$. We call this system a wedge of arcs and denote it by $W$. Since the disk $D$ retracts to a neighborhood of $W$, for many purposes, a consideration for the disk $D$ can be reduced to $W$. For

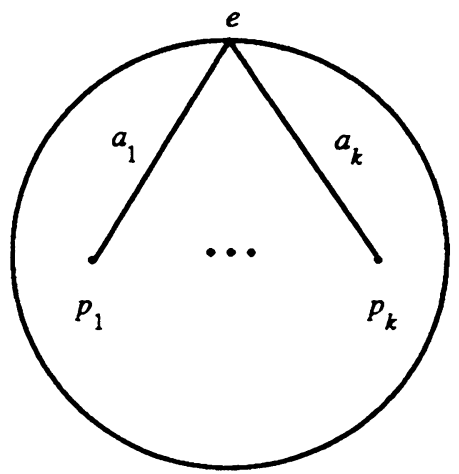

FIGURE 2.1 
example, we have

Lemma 2.5. Suppose $L$ is link-homotopic to $L^{\prime}$, and let $D$ be a $d$-base for $L$. Then there is a d-base $D^{\prime}$ for $L^{\prime}$ such that $(L, D)$ is link-homotopic to $\left(L^{\prime}, D^{\prime}\right)$.

Proof. Ambient isotopy takes $D$ to some other disk $D^{\prime}$ so we need to consider only a crossing change. But such a homotopy occurs in the ball neighborhood of an arc joining two points of one component of the link. We can position the arc to avoid the image of $W$. Then since $D$ deforms to a neighborhood of $W$, we can perform an isotopy of $D$ (keeping the link fixed) so that it avoids the ball in which the homotopy is supported.

Suppose $(L, D)$ is a disk-based link. The image of $W$ will be denoted by the same letter and we call $W$ a $w$-base for $L$ and $(L, W)$ a $w$-based link.

If $(L, W)$ is a $w$-based link, we can have a lot of $d$-bases $D$ for $L$ such that $W \subset D$. But up to isotopy, the only essential difference among these $d$-bases for $L$ is due to some full twistings of the band neighborhoods of the arcs $a_{i}$ in $W$. Such a full twist can be undone by an isotopy which untwists the band. The effect of such an isotopy on the link is a full twisting of two segments of a component of the link which can be eliminated by a link-homotopy. See Figure 2.2 .

We define a link-homotopy of two $w$-based links just like a link-homotopy of two $d$-based links. The only difference is that we now require that the supporting ball of a crossing change have no intersection with $W$. Then the above argument shows that $d$-based link-homotopy is equivalent to $w$-based link-homotopy.

The following proposition and its corollary can be derived from our classification theorem (Theorem 2.9). On the other hand, these can be thought of as prototypes of Theorem 2.9. The method in the proof goes back to [G2] and [Gi].

Proposition 2.6. Suppose $(L, D)$ and $\left(L, D^{\prime}\right)$ are two $d$-based links such that $L$ is link-homotopic to an unlink. Then $(L, D)$ and $\left(L, D^{\prime}\right)$ are link-homotopic.

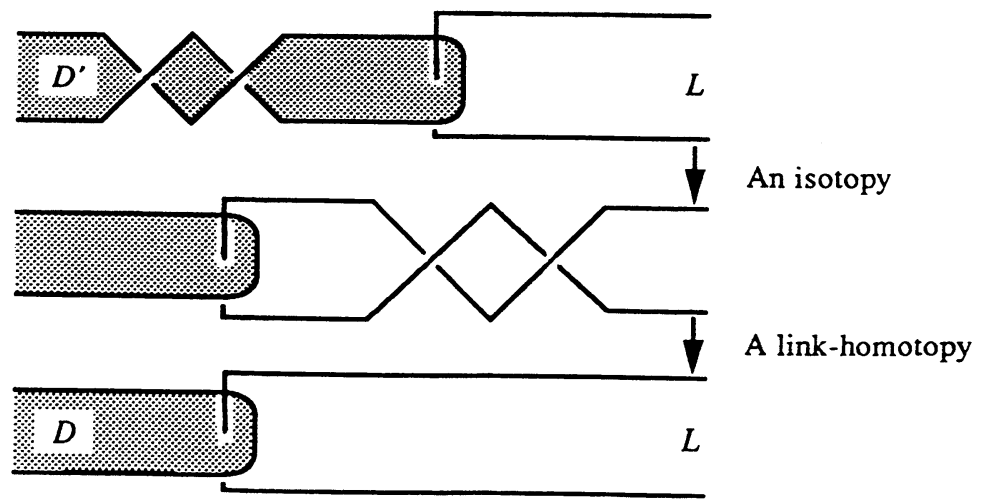

FIGURE 2.2 
Here by an unlink we mean an imbedding $L: \coprod_{i=1}^{k} S_{i}^{1} \rightarrow S^{3}$ which can be extended to an imbedding $\amalg_{i=1}^{k} B_{i}^{2} \rightarrow S^{3}$ where $B_{i}^{2}$ 's are copies of the 2-ball $B^{2}$ and $\partial B^{2}=S^{1}$. Denote the image of $B_{i}^{2}$ by $\Delta_{i}$.

Proof. Equivalently, we consider the $w$-bases of an unlink. For an unlink, there is a standard $w$-base. It is any $w$-base which intersects $\amalg_{i=1}^{k} \Delta_{i}$ only at its endpoints (any two such being isotopic). We need to show only that if $W$ is any $w$-base for an unlink $L$, then $(L, W)$ is link-homotopic to $\left(L, W_{0}\right)$ such that $W_{0}$ is the standard $w$-base for $L$.

Let $a_{i}, i=1, \ldots, k$ be the arcs in $W$. Suppose inductively that $a_{j}$ has no intersection with the interior of $\amalg_{i=1}^{k} \Delta_{i}$ for $j<i$. Let us consider the intersection of $a_{i}$ with the interior of $\Delta_{i}$. Such an intersection can be removed by a link-homotopy of the $w$-based link. First, pull the endpoint $p_{i}$ of $a_{i}$ back towards the base point $e$. This is an isotopy of the $w$-based link. A small neighborhood of $p_{i}$ in $L_{i}$ will be pulled along forming a band passing through the interior of $\Delta_{i}$. Push this band off $\Delta_{i}$. This can be accomplished by a linkhomotopy of the $w$-based link. Finally, isotope the band back along itself. This removes each intersection of $a_{i}$ with the interior of $\Delta_{i}$ at the cost of two self crossings of $L_{i}$. See Figure 2.3. Now it is easy to remove the intersections of $a_{i}$ with $\Delta_{j}, j \neq i$, by isotoping $a_{i} \cup \Delta_{i}$ in the complement of $\bigsqcup_{j \neq i}\left(a_{j} \cup L_{j}\right)$. (This may introduce new intersections of $a_{j}, j>i$, and the interior of $\bigsqcup_{i=1}^{k} \Delta_{i}$.) Thus inductively we have eliminated the intersections of $a_{i}$ and the interior of $\coprod_{i=1}^{k} \Delta_{i}$ by a link-homotopy of the $w$-based link.

Corollary 2.7. Suppose $\sigma$ is a string link such that $\hat{\sigma}$ is link-homotopic to an unlink, then $\sigma=\mathbf{1} \in \mathscr{H}(k)$.

Before continuing, we give a slightly different description of the closure of a string link. Instead of working in $S^{3}$, we will work in a ball $B=B^{3}$ decomposed into three subballs $B_{-}, B_{+}$, and $B_{0}$ with both $B_{-}$and $B_{+}$glued to

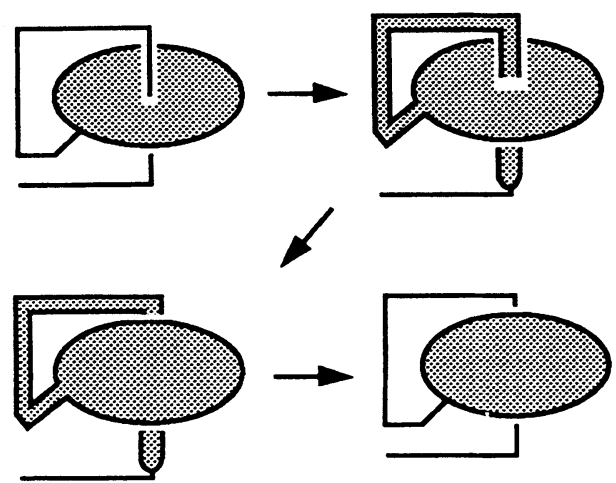

FIGURE 2.3 


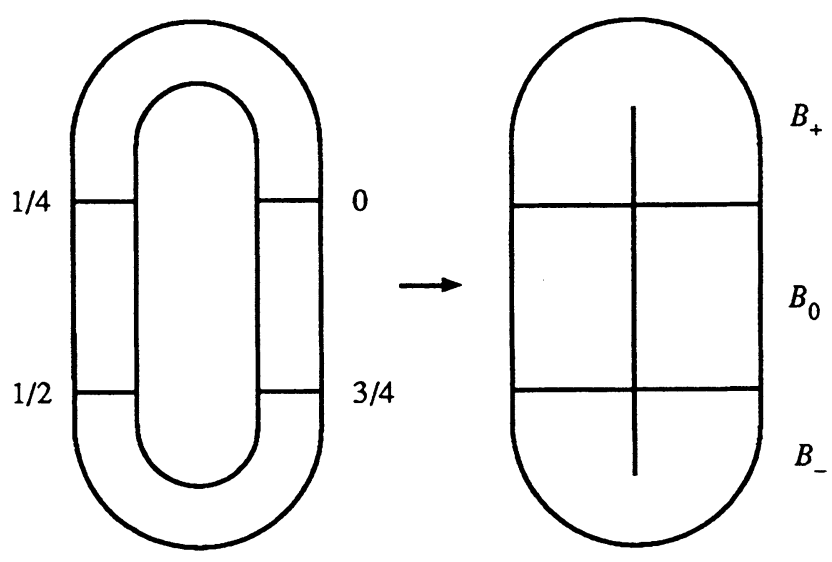

FIGURE 2.4

$B_{0}$ along disks. $B_{0}$ is to be thought of as $D \times I, B_{-}$and $B_{+}$as half balls, and the gluing of $B_{-}$is along the disk $D \times 0$, whereas $B_{+}$is glued along $D \times 1$.

Specifically such a ball can be obtained as an identification space of $D \times I$ where we identify $(z, 0)$ with $(z, 1)$ and perform identifications along $\partial D \times I$ as follows. Let $z=(x, y)$ with $x \leq 0$ and $x^{2}+y^{2}=1$, identify $(z, t)$ with $\left(z, \frac{1}{4}-t(\bmod 1)\right)$ for $t \in[0,1]$. Then $B_{+}, B_{0}$, and $B_{-}$correspond to the product of $D$ with the intervals $\left[0, \frac{1}{4}\right],\left[\frac{1}{4}, \frac{1}{2}\right] \cup\left[\frac{3}{4}, 1\right]$ and $\left[\frac{1}{2}, \frac{3}{4}\right]$ respectively.

Figure 2.4 is a schematic picture of how the identification space is obtained.

Note that $B_{0}$ is the product of the double of the unit disk $D$ along the boundary arc $\left\{(x, y) ; x \leq 0, x^{2}+y^{2}=1\right\}$ with an interval and so can be identified with $D \times I$ with $D$ having $2 k$ distinguished points. The points $p_{i}$ in $D \times \frac{1}{4}$ (also in $D \times \frac{1}{2}$ ) will be labeled with a bar to distinguish them from the points $p_{i}$ in $D \times 0$ (also in $D \times \frac{3}{4}$ ) and a similar convention is made for the loops $x_{i}$ (see Figure 2.5). Note that the orientations of the loops $x_{i}$ are counterclockwise but the orientations of the loops $\bar{x}_{i}$ are clockwise and the points are ordered as

$$
\bar{p}_{k}, \ldots, \bar{p}_{1}, p_{1}, \ldots, p_{k} .
$$

So the boundary curve of the double disk is

$$
\bar{x}_{k}^{-1} \cdots \bar{x}_{1}^{-1} x_{1} \cdots x_{k} \text {. }
$$

Let $\sigma$ be a string link with $k$ strings in $D \times I$. We first identify $D \times I$ with $D \times\left[0, \frac{1}{4}\right]$ by identifying the unit interval $I$ with $\left[0, \frac{1}{4}\right]$. Then we can put $\sigma$ into $B_{+}$since $B_{+}$is an identification space of $D \times\left[0, \frac{1}{4}\right]$ and only different boundary points are identified. In other words, we get a proper imbedding (still denoted by $\sigma$ )

$$
\sigma: \coprod_{i=1}^{k} I_{i} \rightarrow B_{+}
$$




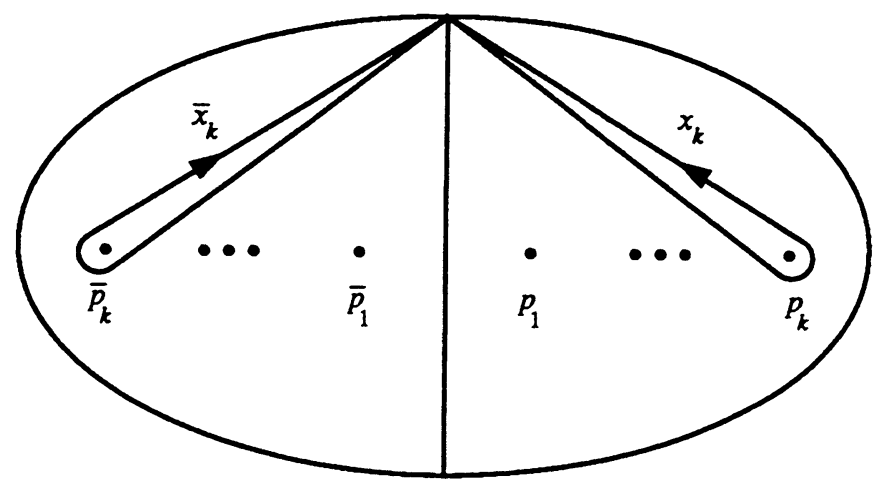

FIGURE 2.5

such that $\left.\sigma\right|_{I_{i}}(0)=p_{i} \in D \times 0$ and $\left.\sigma\right|_{I_{i}}(1)=\bar{p}_{i} \in D \times \frac{1}{4}$. Obviously, any such imbedding is obtained from a string link with $k$ strings.

Let us now construct an action of $\mathscr{H}(2 k)$ on $\mathscr{H}(k)$.

Suppose $\Sigma$ is a string link with $2 k$ strings. We may think of it as a proper imbedding

$$
\Sigma: \coprod_{i=1}^{2 k} I_{i} \rightarrow B_{0}
$$

such that $\left.\Sigma\right|_{I_{i}}(0)=\bar{p}_{k+1-i} \in D \times \frac{1}{2}$ and $\left.\Sigma\right|_{I_{i}}(1)=\bar{p}_{k+1-i} \in D \times \frac{1}{4}$ for $i=$ $1, \ldots, k$ and $\left.\Sigma\right|_{I_{i}}(0)=p_{i-k} \in D \times \frac{3}{4}$ and $\left.\Sigma\right|_{I_{i}}(1)=p_{i-k} \in D \times 0$ for $i=$ $k+1, \ldots, 2 k$. Let $\sigma$ be a string link with $k$ strings. Think of it as a proper imbedding $\amalg_{i=1}^{k} I_{i} \rightarrow B_{+}$. The union of $\sigma$ and $\Sigma$ gives rise to a proper imbedding $\amalg_{i=1}^{k} I_{i} \rightarrow B_{0} \cup B_{+}$. See Figure 2.6. Now identify $B_{0} \cup B_{+}$with $B_{+}$by pushing $B_{0}$ into a collar neighborhood of $B_{+}$. The resulting proper imbedding $\bigsqcup_{i=1}^{k} I_{i} \rightarrow B_{+}$is the image of a string link denoted by $\Sigma \cdot \sigma_{0}$. Notice that as a part of the $i$ th string of the string link $\Sigma \cdot \sigma$, the orientations of $\Sigma\left(I_{i}\right)$ for $i=1, \ldots, k$ have been reversed.

It is easy to see that the above construction gives rise to a well-defined action of $\mathscr{H}(2 k)$ on $\mathscr{H}(k)$.

Lemma 2.8. We have

$$
\left(\Sigma \Sigma^{\prime}\right) \cdot \sigma=\Sigma \cdot\left(\Sigma^{\prime} \cdot \sigma\right)
$$

for $\Sigma, \Sigma^{\prime} \in \mathscr{H}(2 k)$ and $\sigma \in \mathscr{H}(k)$.

Proof. This is obvious from the definition of the action.

We will denote by $\mathscr{S}(k)$ the stabilizer of $1 \in \mathscr{H}(k)$, i.e.,

$$
\mathscr{S}(k)=\{\Sigma \in \mathscr{H}(2 k) ; \Sigma \cdot \mathbf{1}=\mathbf{1} \in \mathscr{H}(k)\} .
$$

By Lemma 2.8 , we see that $\mathscr{S}(k)$ is a group. 


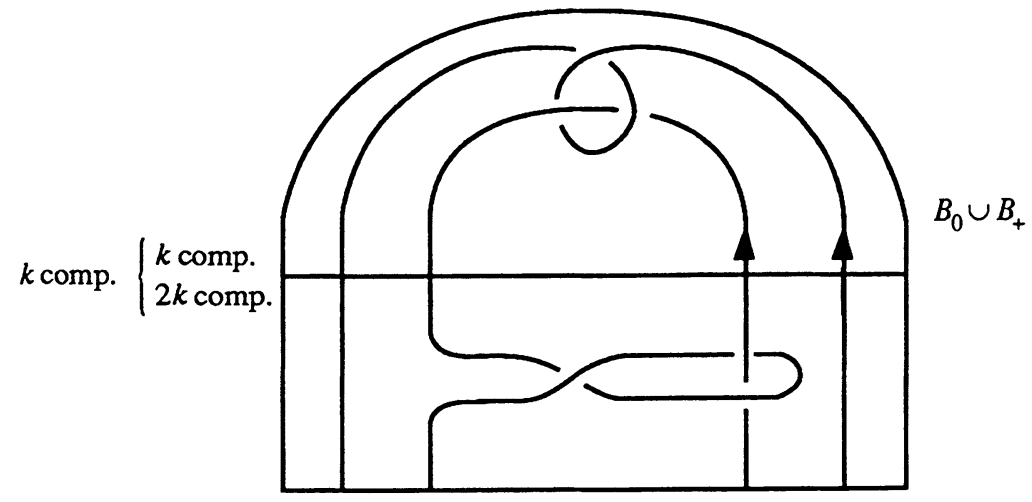

FIGURE 2.6

Theorem 2.9. Suppose $\sigma_{1}, \sigma_{2} \in \mathscr{H}(k)$, then $\hat{\sigma}_{1}$ and $\hat{\sigma}_{2}$ are link-homotopic if and only if there is a $\Sigma \in \mathscr{S}(k)$ such that $\Sigma \cdot \sigma_{1}=\sigma_{2}$.

Proof. Let $B=B_{-} \cup B_{0} \cup B_{+} \subset S^{3}$. Let us identify $D \times I$ with $D \times\left[\frac{1}{2}, \frac{3}{4}\right]$. We will still use 1 to denote the composition of the imbedding $1: \coprod_{i=1}^{k} I_{i} \rightarrow D \times I$ with the quotient map $D \times I \rightarrow B_{-}$. Then the union of the string link 1 in $B_{-}$and the string link $\Sigma \cdot \sigma$ in $B_{0} \cup B_{+}$gives us a link in $B \subset S^{3}$. This link is $\widehat{\Sigma \cdot \sigma}$. If we choose $\Sigma$ to be the trivial string link with $2 k$ strings, then the resulting link in the above construction is $\hat{\sigma}$. Notice that the ordering and orientation of the components of $\hat{\sigma}$ coincide with the ordering and orientation of the strings of $\sigma$ in the above construction. We will regard this construction as the canonical way to close a string link up to get a link.

Suppose $\Sigma \in \mathscr{S}(k)$. Notice that there is an involution on $B$ which maps $B_{+}$to $B_{-}$and leaves $D \times \frac{3}{8} \cup D \times \frac{7}{8}$ fixed. The image of the union of 1 in $B_{-}$and $\Sigma$ in $B_{0}$ with orientation reversed is the same as $\Sigma^{-1} \cdot 1$ which is link-homotopic to the trivial string link by our assumption. So the union of 1 in $B_{-}$and $\Sigma$ in $B_{0}$, thought of as a string link, is also link-homotopic to the trivial one. Thus we have proved that if $\Sigma \in \mathscr{S}(k)$ and $\sigma \in \mathscr{H}(k)$, then $\widehat{\Sigma \cdot \sigma}$ is link-homotopic to $\hat{\sigma}$.

Let us now consider the other direction of our theorem. Since the set of link-homotopy classes of string links is in one-one correspondence with the set of link-homotopy classes of $d$-based links (Corollary 2.4), to study the relation between two string links $\sigma_{1}$ and $\sigma_{2}$ when $\hat{\sigma}_{1}$ and $\hat{\sigma}_{2}$ are link-homotopic, we need to study only the relation between two $d$-based links $\left(L_{1}, D_{1}\right)$ and $\left(L_{2}, D_{2}\right)$ when $L_{1}$ and $L_{2}$ are link-homotopic. By Lemma 2.5, $\left(L_{1}, D_{1}\right)$ is link-homotopic to $\left(L_{2}, D_{2}^{\prime}\right)$, so we need to study only the relation between two string links determined by two different $d$-bases of a single link.

Let $\sigma$ be a string link, which we assume is in $B_{+}$. We close it up canonically in $B \subset S^{3}$ and pick $D=D_{1-\varepsilon} \times \frac{5}{8}$ to be the standard $d$-base for $\hat{\sigma}$. Here $D_{1-\varepsilon}$ is the disk with radius $1-\varepsilon$ and $p_{i} \in D_{1-\varepsilon}$ for $i=1, \ldots, k$. Up to 


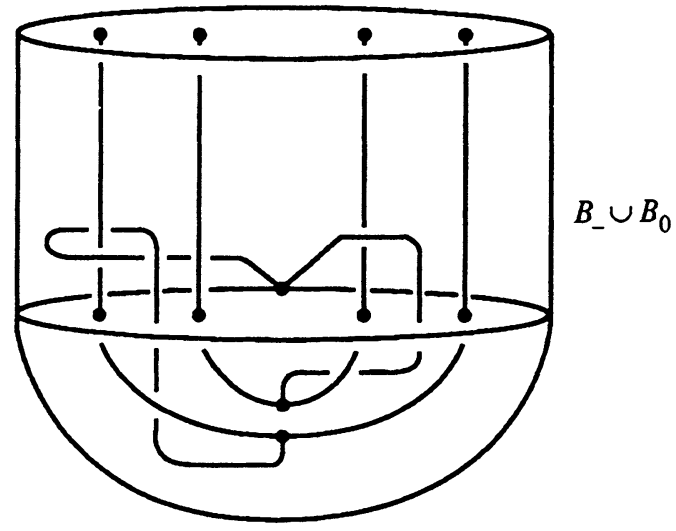

FIGURE 2.7

link-homotopy, we can assume $\sigma$ in $B_{+}$is a pure braid (see the remark after the proof of Lemma 1.8). Then the complement of $\sigma$ in $B_{+}$has a product structure. Consequently, any other $d$-base $D^{\prime}$ of $\hat{\sigma}$ can be isotoped into the interior of $B_{-} \cup B_{0}$ keeping $\hat{\sigma}$ fixed. See Figure 2.7 in which we have drawn schematically the image of the corresponding $w$-base.

Suppose $D_{0}^{\prime}$ and $D_{1}^{\prime}$ are the top disk and bottom disk of a small product neighborhood $N$ of $D^{\prime}$ in the interior of $B_{-} \cup B_{0}$ such that $\hat{\sigma} \cap N$ is a trivial string link with $k$ strings. Now perform an isotopy of $B_{-} \cup B_{0}$ keeping the boundary fixed, which takes $\left(N, D_{0}^{\prime}, D_{1}^{\prime}\right)$ to

$$
\left(D_{1-\varepsilon} \times\left[\frac{1}{2}, \frac{3}{4}\right], D_{1-\varepsilon} \times \frac{1}{2}, D_{1-\varepsilon} \times \frac{3}{4}\right) \subset\left(B_{+}, D \times \frac{1}{2}, D \times \frac{3}{4}\right)
$$

and so that the intersection of the image of $\hat{\sigma}$ with $B_{-}$is a trivial string link with $k$ strings. It is clear that the intersection of the image of $\hat{\sigma}$ with $B_{0}$ is a string link $\Sigma$ with $2 k$ strings.

Observe that the part of the image of $\hat{\sigma}$ in $B_{0} \cup B_{+}$is the string link determined by the $d$-based link $\left(\hat{\sigma}, D^{\prime}\right)$, and moreover it can be written as $\Sigma \cdot \sigma$. So the only thing we need to show is $\Sigma \in \mathscr{S}(k)$. But by construction, after reflecting $B_{0} \cup B_{+}$into $B_{-} \cup B_{0}$ by the involution on $B$ mentioned above, $\Sigma^{-1} \cdot \mathbf{1}$ is isotopic to the trivial string link $\hat{\sigma} \cap\left(B_{-} \cup B_{0}\right)$. So $\Sigma^{-1} \in \mathscr{S}(k)$ and consequently $\Sigma \in \mathscr{S}(k)$. This proves Theorem 2.9.

We conclude this section with a study of the group $\mathscr{S}(k)$ and its action on $\mathscr{H}(k)$. We are led to a Markov-type theorem, which was conjectured by Levine more that ten years ago.

In Figure 2.8 are some planar projections of elements in $\mathscr{S}(k)$. We use the following notation there. The first $k$ strings are named by $\bar{k}, \ldots, \overline{1}$ in that order and the last $k$ strings are named $1, \ldots, k$ in that order. All the strings are oriented upward. The base point is behind the picture.

Note that if $\Sigma \in \mathscr{H}(2 k)$ and $\sigma \in \mathscr{H}(k)$, the $i$ th and $\bar{i}$ th strings of $\Sigma$ become part of the $i$ th string of $\Sigma \cdot \sigma$. Hence if $\Sigma$ is changed by crossings of 

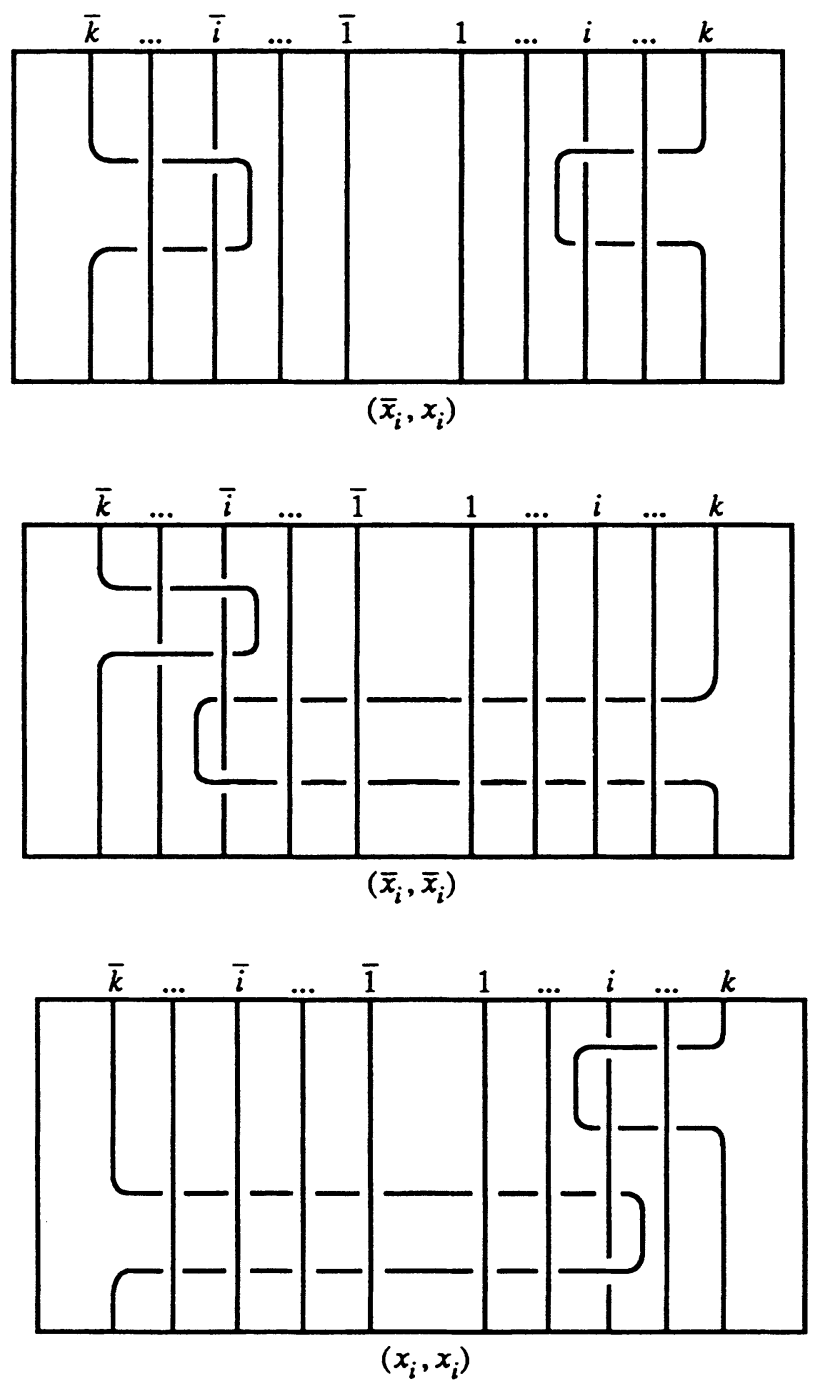

FIGURE 2.8

these pairs of strings, the link-homotopy class of $\Sigma \cdot \sigma$ remains unchanged. Thus the action of $\mathscr{H}(2 k)$ factors through a quotient, denoted by $\mathscr{H}^{*}(2 k)$, suitably defined. The work in $\S 1$ generalizes to this setting, in particular yielding a split short exact sequence:

$$
1 \rightarrow R F^{*}(2 k-2) \times R F^{*}(2 k-2) \rightarrow \mathscr{H}^{*}(2 k) \rightarrow \mathscr{H}^{*}(2 k-2) \rightarrow 1 .
$$

Some remarks about the above exact sequence are in order. First of all, the map $\mathscr{H}^{*}(2 k) \rightarrow \mathscr{H}^{*}(2 k-2)$ is given by omitting the $k$ th and $\bar{k}$ th strings. 
Next, the group $R F^{*}(2 k-2)$ is obtained from

$$
R F(2 k-2)=R F\left(\bar{x}_{k-1}, \ldots, \bar{x}_{1}, x_{1}, \ldots, x_{k-1}\right)
$$

by adding relations saying that $x_{i}$ commutes with conjugates of $\bar{x}_{i}$. Finally, a pair of words $(h, g)$ with $g, h \in R F^{*}(2 k-2)$ gives rise to a string link with $2 k$ strings obtained from representing $g$ and $h$ as the $k$ th string and $\bar{k}$ th string in the complement of a trivial string link with $2 k-2$ strings.

Corresponding to the above short exact sequence for $\mathscr{H}^{*}(2 k)$ is one for $\mathscr{S}^{*}(k)$, the image of $\mathscr{S}(k)$ :

$$
1 \rightarrow \mathscr{K} \rightarrow \mathscr{S}^{*}(k) \rightarrow \mathscr{S}^{*}(k-1) \rightarrow 1 .
$$

We define

$$
\begin{aligned}
\eta: R F^{*}(2 k-2) \rightarrow R F(k-1) & =R F\left(x_{1}, \ldots, x_{k-1}\right) \\
\eta\left(x_{i}\right)=\eta\left(\bar{x}_{i}\right)=x_{i}, \quad i & =1, \ldots, k-1 .
\end{aligned}
$$

Lemma 2.10. For $(h, g) \in R F^{*}(2 k-2) \times R F^{*}(2 k-2) \subset \mathscr{H}^{*}(2 k),(h, g) \in \mathscr{K}$ if and only if $\eta(h)=\eta(g)$.

Proof. Note that $(h, g) \cdot \mathbf{1}=\eta\left(h g^{-1}\right) \in R F(k-1) \subset \mathscr{H}(k)$. So $(h, g) \in \mathscr{K}$ if and only if $\eta(h)=\eta(g)$.

For example, the string links in Figure 2.8 all represent elements in $\mathscr{K}$.

Lemma 2.11. $\mathscr{K}$ is generated by

$$
\left\{\left(\bar{x}_{i}, x_{i}\right),\left(\bar{x}_{i}, \bar{x}_{i}\right),\left(x_{i}, x_{i}\right) ; i=1, \ldots, k-1\right\} .
$$

Proof. Let $\mathscr{K}_{0}$ be the subgroup generated by these elements. Then $\mathscr{K}_{0} \subset \mathscr{K}$. One has that $\left(x_{i}^{-1} \bar{x}_{i}, 1\right),\left(1, x_{i}^{-1} \bar{x}_{i}\right)$, and $\left(x_{i}, \bar{x}_{i}\right)$ are all in $\mathscr{K}_{0}$.

Let $\tilde{h}$ be the word obtained from a word $h$ by replacing $x_{i}\left(\bar{x}_{i}\right)$ in $h$ by $\bar{x}_{i}$ $\left(x_{i}\right)$. Both $(\tilde{h}, h)$ and $(h, \tilde{h})$ belong to $\mathscr{K}_{0}$ for any $h \in R F\left(x_{1}, \ldots, x_{k-1}\right)$. And we also have $\widetilde{h^{-1}}=\tilde{h}^{-1}$. Hence

$$
(\tilde{h}, h)\left(1, x_{i}^{-1} \bar{x}_{i}\right)\left(\tilde{h}^{-1}, h^{-1}\right)=\left(1, h x_{i}^{-1} \bar{x}_{i} h^{-1}\right) \in \mathscr{K}_{0} .
$$

Now if $(1, g) \in \mathscr{K}$, then $\eta(g)=1$, i.e., $g$ is a product of conjugates of $x_{i}^{-1} \bar{x}_{i}$. So by the above discussion, $(1, g) \in \mathscr{K}_{0}$.

For $(h, g) \in \mathscr{K}$, we have $\eta(h)=\eta(g)$. Then since

$$
(h, g)=(h, \tilde{h})\left(1, \tilde{h}^{-1} g\right)
$$

and

$$
\eta(\tilde{h})=\eta(h),
$$

we get $(h, g) \in \mathscr{K}_{0}$ and hence $\mathscr{K}_{0} \subset \mathscr{K}$. So $\mathscr{K}_{0}=\mathscr{K}$.

Let us now consider the actions of $\left(\bar{x}_{i}, x_{i}\right),\left(\bar{x}_{i}, \bar{x}_{i}\right)$, and $\left(x_{i}, x_{i}\right)$ on $\mathscr{H}(k)$. Let $\theta \in \mathscr{H}(k-1)$ and $g \in R F\left(x_{1}, \ldots, x_{k-1}\right)$. Figure 2.9 shows the action of $\left(\bar{x}_{i}, \bar{x}_{i}\right)$ on $\theta g \in \mathscr{H}(k)$. One finds

$$
\left(\bar{x}_{i}, \bar{x}_{i}\right) \cdot \theta g=\theta x_{i} g x_{i}^{-1}=\theta g^{x_{i}} .
$$




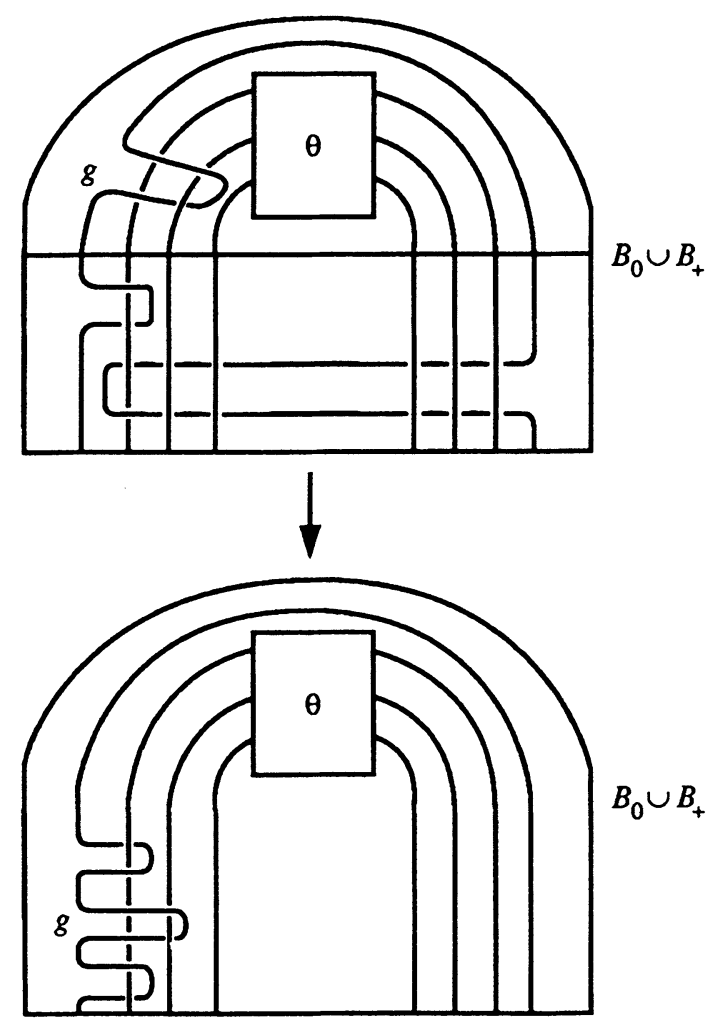

FigURE 2.9

Similarly, we have

$$
\left(x_{i}, x_{i}\right) \cdot g \theta=x_{i} g x_{i}^{-1} \theta
$$

It follows that

$$
\begin{aligned}
\left(x_{i}, x_{i}\right) \cdot \theta g & =\left(x_{i}, x_{i}\right) \cdot \theta g \theta^{-1} \theta=x_{i} \theta g \theta^{-1} x_{i}^{-1} \theta \\
& =\theta\left(\theta^{-1} x_{i} \theta\right) g\left(\theta^{-1} x_{i}^{-1} \theta\right)=\theta g^{\theta^{-1} x_{i} \theta} .
\end{aligned}
$$

So both $\left(\bar{x}_{i}, \bar{x}_{i}\right)$ and $\left(x_{i}, x_{i}\right)$ act on $\mathscr{H}(k)$ by "partial conjugation" which we now define.

Definitin 2.12. Let $\sigma \in \mathscr{H}(k)$ be decomposed as $\theta g$ corresponding to the decomposition $\mathscr{H}(k)=\mathscr{H}(k-1) \ltimes R F(k-1)$ obtained from the map $\mathscr{H}(k) \rightarrow$ $\mathscr{l}(k-1)$ given by omission of the $i$ th string. A partial conjugate of $\sigma$ is an element of $\mathscr{H}(k)$ of the form $\theta g^{h}$ with $h \in R F(k-1)$. 
Notice that $\left(\bar{x}_{i}, x_{i}\right)$ acts on $\mathscr{H}(k)$ by conjugation by $x_{i} \in R F(k-1) \subset$ $\mathscr{H}(k)$. We then have

Theorem 2.13. Let $\sigma, \sigma^{\prime} \in \mathscr{H}(k)$. Then the closures of $\sigma$ and $\sigma^{\prime}$ are linkhomotopic as ordered, oriented links if and only if there is sequence $\sigma=\sigma_{0}, \sigma_{1}$, $\ldots, \sigma_{n}=\sigma^{\prime}$ of elements of $\mathscr{H}(k)$ such that $\sigma_{j+1}$ is either a conjugate or a partial conjugate of $\sigma_{j}$.

Proof. $\mathscr{S}^{*}(k)$ is generated by the kernels of the various quotient maps $\mathscr{S}^{*}(k) \rightarrow \mathscr{S}^{*}(k-1)$. Each such kernel is generated by elements which act on $\mathscr{H}(k)$ by conjugation or partial conjugation. Moreover, these generators are also generators for the full group generated by conjugation and partial conjugation, since $R F(k-1)$ is generated by the $x_{i}$ and since $\mathscr{H}(k)$ is generated by the various subgroups $R F(k-1) \subset \mathscr{H}(k)$.

Remark. It is geometrically clear that conjugate or partially conjugate string links determine link-homotopic closed links. The former holds because cyclically permuted products of string links yield the same closed link. The latter holds because partial conjugation corresponds to conjugating one component of the closed link in the fundamental group of the complement of the others.

\section{MILNOR'S INTEGER INVARIANTS}

According to Milnor, the link-homotopy classes of ordered, oriented links with $k$ components can "roughly" be described by

$$
\sum_{m=2}^{k}(m-2) !\left(\begin{array}{l}
k \\
m
\end{array}\right)
$$

integers. Our string link description gives a precise interpretation of these invariants. The rank of the torsion-free nilpotent group $\mathscr{H}(k)$ is exactly this number. In fact, the rank of the free abelian group $\mathscr{H}(k)_{m-1} / \mathscr{H}(k)_{m}$ is $(m-2) !\left(\begin{array}{l}k \\ m\end{array}\right)$ and Milnor's rough construction of links closely parallels the precise decomposition

$$
\mathscr{H}(k)=(\cdots(R F(1) \ltimes R F(2)) \ltimes \cdots) \ltimes R F(k-1) .
$$

Thus Milnor's integers, while not link-homotopy invariants of links, are linkhomotopy invariants of string links.

The true link-homotopy invariants of links are invariants of the orbit space $\mathscr{H}(k) / \mathscr{S}^{*}(k)$. However, the nilpotent structure of $\mathscr{H}(k)$ and the complicated nature of the action of $\mathscr{S}^{*}(k)$ conspire to make it difficult, if not impossible, to find a complete set of invariants.

In [M1], Milnor did obtain link-homotopy invariants of links by taking the above integer invariants modulo indeterminancies given by greatest common divisors of lower order integer invariants. In [M2], these indeterminancies were replaced by smaller ones. (It is still not known if they are actually smaller, a problem posed at the 1982 Sussex conference. See [F], problem list.) However, these indeterminancies are not sharp enough to classify links. Computations of 
Vogel using our results have shown that any refinement of the indeterminancies of the integer invariants cannot be done individually. In order to get a group structure on the secondary invariants of 4-component links, one needs to extend all four secondary $\bar{\mu}$-invariants simultaneously.

At this point, we prefer to rely on Theorem 2.13. Note that the conjugacy problem of a nilpotent group is solvable (see [Ba]). Therefore, there are algorithms to separately decide if two elements in $\mathscr{H}(k)$ are conjugate or partially conjugate.

Theorem 3.1. There is an algorithm which will decide whether or not two elements in $\mathscr{H}(k)$ are equivalent under the equivalence relation generated by conjugation and partial conjugation.

This result is somewhat remarkable, for often it is the case that an equivalence relation generated by two others is not recursive.

In this section, we will use $s(\cdot)$ to denote the action of $s \in \mathscr{S}^{*}(k)$ on $\mathscr{H}(k)$.

The key to understanding why Theorem 3.1 is true is the observation that even though conjugation and partial conjugation are different (the former acts by automorphisms whereas the latter does not), they have two properties in common.

Lemma 3.2. For $s \in \mathscr{S}^{*}(k)$ and $\xi \in \mathscr{H}(k)_{i}$, one has

(1) $s(\mathbf{1})=\mathbf{1}$;

(2) $s(\xi \sigma) \equiv \xi s(\sigma) \bmod \mathscr{H}(k)_{i+1}$ for any $\sigma \in \mathscr{H}(k)$.

Proof. The first is the defining property of $\mathscr{S}^{*}(k)$. Let us consider the second property. For conjugation, one has

$$
s(\xi \sigma)=s(\xi) s(\sigma) \text { and } s(\xi) \equiv \xi \bmod \mathscr{H}(k)_{i+1} .
$$

For partial conjugation, set $\xi=\theta^{\prime} g^{\prime}$ and $\sigma=\theta g$ for $\theta, \theta^{\prime} \in \mathscr{H}(k-1)$ and $g, g^{\prime} \in R F(k-1)$. Then for some $h \in R F(k-1)$, we have

$$
s(\xi \sigma)=\theta^{\prime} \theta\left(\theta^{-1} g^{\prime} \theta\right)^{h} g^{h}
$$

whereas

$$
\xi s(\sigma)=\theta^{\prime} \theta\left(\theta^{-1} g^{\prime} \theta\right) g^{h} .
$$

Now $\xi \in \mathscr{H}(k)_{i}$, so $g^{\prime} \in R F(k-1)_{i}$ and $\theta^{-1} g^{\prime} \theta \in R F(k-1)_{i}$. Thus

$$
\left(\theta^{-1} g^{\prime} \theta\right)^{h} \equiv \theta^{-1} g^{\prime} \theta \quad \bmod R F(k-1)_{i+1} \subset \mathscr{H}(k)_{i+1} \text {, }
$$

or

$$
s(\xi \sigma) \equiv \xi s(\sigma) \bmod \mathscr{H}(k)_{i+1} .
$$

Now let $\mathscr{S}$ be a group acting on a group $\mathscr{H}$ and satisfying

(1) $s(\mathbf{1})=\mathbf{1}$; and

(2) $s(\xi \sigma) \equiv \xi s(\sigma) \bmod \mathscr{H}_{i+1}$ for $\xi \in \mathscr{H}_{i}$ and $\sigma \in \mathscr{H}$. 
Then the action of $\mathscr{S}$ passes to an action on $\mathscr{H} / \mathscr{H}_{i}$ commuting with the action of $\mathscr{H}_{i} / \mathscr{H}_{i+1}$ given by multiplication. In particular, each fiber of the projection of the orbit spaces

$$
\frac{\mathscr{H} \mid \mathscr{H}_{i+1}}{\mathscr{S}} \rightarrow \frac{\mathscr{H} \mid \mathscr{H}_{i}}{\mathscr{S}}
$$

has a transitive action of $\mathscr{H}_{i} / \mathscr{H}_{i+1}$ and hence the structure of an affine space of a quotient group of $\mathscr{H}_{i} / \mathscr{H}_{i+1}$.

The above discussion leads to an "obstruction theory" picture. Suppose $\sigma, \sigma^{\prime} \in \mathscr{H}$. We check inductively whether they agree in $\left(\mathscr{H} / \mathscr{H}_{i}\right) / \mathscr{S}$. Assume they do. Then there is a well-defined "difference" coset $d_{i}\left(\sigma, \sigma^{\prime}\right) \subset \mathscr{H}_{i} / \mathscr{H}_{i+1}$ such that $\sigma$ and $\sigma^{\prime}$ agree in $\left(\mathscr{H} / \mathscr{H}_{i+1}\right) / \mathscr{S}$ if and only if $d_{i}\left(\sigma, \sigma^{\prime}\right)$ contains zero. This leads to the problem of trying to compute these cosets.

Let us use the same notation to denote the induced action of $\mathscr{S}$ on $\mathscr{H} / \mathscr{H}_{i}$. For $\sigma \in \mathscr{H}$, we will denote by $\sigma_{i}$ its image in $\mathscr{H} / \mathscr{H}_{i}$.

Define

$$
\mathscr{S}_{\sigma_{i}}=\left\{s \in \mathscr{S} ; s(\sigma) \equiv \sigma \bmod \mathscr{H}_{i}\right\} .
$$

In other words, $\mathscr{S}_{\sigma_{i}}$ is the stablizer of $\sigma_{i}$ in $\mathscr{H} / \mathscr{H}_{i}$. If $s \in \mathscr{S}_{\sigma_{i}}$, then the "difference"

belongs to $\mathscr{H}_{i} / \mathscr{H}_{i+1}$.

$$
d_{i}^{\sigma}(s)=s\left(\sigma_{i+1}\right) \sigma_{i+1}^{-1}
$$

Lemma 3.3. The map $s \mapsto d_{i}^{\sigma}(s)$ is a homomorphism

$$
d_{i}^{\sigma}: \mathscr{S}_{\sigma_{i}} \rightarrow \mathscr{H}_{i} / \mathscr{H}_{i+1}
$$

and its kernel is $\mathscr{S}_{\sigma_{i+1}}$.

Proof. Suppose $s_{1}, s_{2} \in \mathscr{S}_{\sigma_{i}}$, by property (2) of $\mathscr{S}$ and the fact that $\mathscr{H}_{i} / \mathscr{H}_{i+1}$ is an abelian group. We get

$$
\begin{aligned}
d_{i}^{\sigma}\left(s_{1}\right) d_{i}^{\sigma}\left(s_{2}\right) & =s_{1}\left(\sigma_{i+1}\right) \sigma_{i+1}^{-1} s_{2}\left(\sigma_{i+1}\right) \sigma_{i+1}^{-1} \\
& =s_{2}\left(\sigma_{i+1}\right) \sigma_{i+1}^{-1} s_{1}\left(\sigma_{i+1}\right) \sigma_{i+1}^{-1} \\
& =s_{1}\left(s_{2}\left(\sigma_{i+1}\right) \sigma_{i+1}^{-1} \sigma_{i+1}\right) \sigma_{i+1}^{-1} \\
& =s_{1}\left(s_{2}\left(\sigma_{i+1}\right)\right) \sigma_{i+1}^{-1} \\
& =d_{i}^{\sigma}\left(s_{1} s_{2}\right) .
\end{aligned}
$$

So $d_{i}^{\sigma}$ is a homomorphism. The second claim is clear from the definition.

Now suppose $\sigma, \sigma^{\prime} \in \mathscr{H}$ such that $\sigma_{i}=\sigma_{i}^{\prime}$. Define the "difference"

$$
d\left(\sigma_{i+1}, \sigma_{i+1}^{\prime}\right)=\sigma_{i+1}^{\prime} \sigma_{i+1}^{-1} \in \mathscr{H}_{i} / \mathscr{H}_{i+1}
$$

and define the "difference coset"

$$
d_{i}\left(\sigma, \sigma^{\prime}\right)=d\left(\sigma_{i+1}, \sigma_{i+1}^{\prime}\right) d_{i}^{\sigma}\left(\mathscr{S}_{\sigma_{i}}\right) \subset \mathscr{H}_{i} / \mathscr{H}_{i+1} .
$$


Lemma 3.4. There is an $s \in \mathscr{S}$ such that $s\left(\sigma_{i+1}\right)=\sigma_{i+1}^{\prime}$ if and only if $d\left(\sigma_{i+1}, \sigma_{i+1}^{\prime}\right)$ belongs to the image of $d_{i}^{\sigma}$ or 0 belongs to the coset $d_{i}\left(\sigma, \sigma^{\prime}\right)$.

Proof. We need to notice only that if such an $s \in \mathscr{S}$ exists, we must have $s \in \mathscr{S}_{\sigma_{i}}$ since we have assumed that $\sigma_{i}=\sigma_{i}^{\prime}$. Then the lemma follows directly from the definition.

Assuming there is an $s \in \mathscr{S}$ such that $s\left(\sigma_{i+1}\right)=\sigma_{i+1}^{\prime}$, then we replace $\sigma^{\prime}$ by $s^{-1}\left(\sigma^{\prime}\right)$. For this new $\sigma^{\prime}$, we have $\sigma_{i+1}=\sigma_{i+1}^{\prime}$ so we can continue to search for some $s \in \mathscr{S}$ such that $s\left(\sigma_{i+2}\right)=\sigma_{i+2}^{\prime}$.

In order for the above procedure to be effective, we need to be able to compute $d_{i}^{\sigma}$, in particular to know $\mathscr{S}_{\sigma_{i}}$ and its action, or at least a finite set of generators for $\mathscr{S}_{\sigma_{i}}$ whose action on $\mathscr{H}^{i}$ we can compute. In the case that $\mathscr{H}$ is a torsionfree nilpotent group, to say the action on $\mathscr{H}$ is computable means we know how the coefficients of an element change under the action with respect to a fixed basis for $\mathscr{H}$. Suppose inductively such generators $s_{j}$ exist. Let $\mathscr{F}_{i}$ be the free group on these generators. Since $\mathscr{H}_{i} / \mathscr{H}_{i+1}$ is abelian, the composite map

$$
D_{i}^{\sigma}: \mathscr{F}_{i} \rightarrow \mathscr{H}_{i} / \mathscr{H}_{i+1}
$$

factors through the abelianization to give

$$
\bar{D}_{i}^{\sigma}: \mathscr{F}_{i} /\left(\mathscr{F}_{i}\right)_{2} \rightarrow \mathscr{H}_{i} / \mathscr{H}_{i+1} .
$$

In our case, $\mathscr{H}_{i} / \mathscr{H}_{i+1}$ is free abelian, so $\bar{D}_{i}^{\sigma}$ is just an integer matrix which is diagonalizable. Hence it can be decided if $d\left(\sigma_{i+1}, \sigma_{i+1}^{\prime}\right) \in \mathscr{H}_{i} / \mathscr{H}_{i+1}$ belongs to the image of $\bar{D}_{i}^{\sigma}$ and if it does, construct a preimage. This will be a linear combination of $\bar{s}_{j}$ in $\mathscr{F}_{i} /\left(\mathscr{F}_{i}\right)_{2}$ and the corresponding word in $s_{j}$ will be an $s \in \mathscr{S}$ with $s\left(\sigma_{i+1}\right)=\sigma_{i+1}^{\prime}$. Moreover, since the action of $s_{j}$ is computable, $s^{-1}\left(\sigma^{\prime}\right)$ can be computed, since $s^{-1}$ is a word in the $s_{j}$.

Again, since the matrix of $\bar{D}_{i}^{\sigma}$ is diagonalizable, a basis of the kernel of $\bar{D}_{i}^{\sigma}$ can be given in terms of linear combinations of $\bar{s}_{j}$ and hence the corresponding words in $\mathscr{F}_{i}$ together with $\left(\mathscr{F}_{i}\right)_{2}$ will generate $\mathscr{S}_{\sigma_{i+1}}$. If $\mathscr{S}$ is nilpotent of class $n$ (as it is in our case), then $\left(\mathscr{F}_{i}\right)_{n}$ will automatically map trivially to $\mathscr{S}_{\sigma_{i+1}}$. Hence we will get a finite set of generators by taking the basic commutators of length $\leq n$ in $\left(\mathscr{F}_{i}\right)_{2}$. Since all of these will be expressible in terms of words in the $s_{j}$, their action will be computable.

This completes the algorithm and the proof of Theorem 3.1. Note that $\mathscr{S}^{*}(k)$, and hence the group generated by conjugation and partial conjugation, is nilpotent of class $k-1$. Conjugation can be generated by $\left(k^{2}-k\right) / 2$ generators, whereas each of the $k$ partial conjugations can be generated by $k-1$ generators, for a total of $3 k(k-1) / 2$ generators. 
We conclude this section with some ancilliary remarks. Note that we have achieved something more with the above algorithm. For any $\sigma$, we have constructed a set of generators for its stabilizer $\mathscr{S}_{\sigma}^{*}$, since $\sigma_{k}=\sigma$.

The stabilizer $\mathscr{S}_{\sigma}^{*}$ has geometric meaning. If $\Sigma \in \mathscr{S}_{\sigma}^{*}$, we can construct two link-homotopies from $\widehat{\Sigma \cdot \sigma}$ to $\hat{\sigma}$. One link-homotopy is the extension of the link-homotopy from $\Sigma \cdot \sigma$ to $\sigma$, and the other is the extension of the linkhomotopy from (the reflection of) $\Sigma^{-1} \cdot 1$ to 1 in $B_{-} \cup B_{0}$ which keeps $\sigma$ fixed. The union of these two gives a link-homotopy from $\hat{\sigma}$ to itself. Now a selflink-homotopy of a link $L$ produces an automorphism of Milnor's link group $\mathscr{G}(L)=R \pi_{1}\left(\mathscr{S}^{3} \backslash L\right)$. Levine calls this a geometric automorphism [Le2]. His study of the geometric automorphism group for three component link groups led him to classify four component links. A link $L$ with an additional component $\alpha$ added, written $L \cup \alpha$, is link-homotopic to $L \cup \beta$ if and only if $\alpha$ and $A(\beta)$ are conjugate in $\mathscr{G}(L)$ for some geometric automorphism $A$.

Our work began by attempting to understand the geometric automorphisms by "lifting" them to $R F(k)$. (This is what led us to consider automorphisms in the first place and eventually to string links!) Now $d$-bases (or $w$-bases) of a link $L$ give rise to maps $F(k) \rightarrow \pi_{1}\left(S^{3} \backslash L\right)$ and since a self-link-homotopy of a link takes one base to another, the corresponding geometric automorphism does in fact lift to an automorphism of $R F(k)$ obtained as follows. The link $\widehat{\Sigma \cdot \sigma}$ has two $d$-bases corresponding to the disks $D_{0}=D \times 0$ and $D_{3 / 4}=D \times \frac{3}{4}$. Since both $D_{0}$ and $D_{3 / 4}$ lie in $B_{-} \cup B_{0}$ containing the string link $\Sigma^{-1} \cdot 1$, the diagram

$$
\left(D_{0} \backslash\left\{p_{i}\right\}\right) \rightarrow\left(\left(B_{-} \cup B_{0}\right) \backslash \Sigma^{-1} \cdot \mathbf{1}\right) \leftarrow\left(D_{3 / 4} \backslash\left\{p_{i}\right\}\right)
$$

yields an automorphism $\phi_{\Sigma}$ of $R F(k)$. Such an automorphism satisfies (1) $\phi\left(x_{i}\right)$ is conjugate to $x_{i}$, but not in general (2) $\phi\left(x_{1} \cdots x_{k}\right)=x_{1} \cdots x_{k}$. Thus these automorphisms are "special" but not in general braid-like.

One can check that the correspondence $\Sigma \mapsto \phi_{\Sigma}$ is a homomorphism from $\mathscr{S}^{*}(k)$ to $\mathrm{Aut}_{s}(R F(k))$, the special automorphism group. Every special automorphism can be realized by a base change of the unlink, and Proposition 2.6 shows that such a base change can always be obtained by a self-link-homotopy in the case of an unlink. Thus the geometric automorphism group of an unlink equals the special automorphism group and the map $\mathscr{S}^{*}(k) \rightarrow \operatorname{Aut}_{s}(R F(k))$ is onto.

More generally, every element of $\mathscr{S}_{\sigma}^{*}$ maps to an automorphism which descends to an automorphism of $\mathscr{G}(\hat{\sigma})=R \pi_{1}\left(S^{3} \backslash \hat{\sigma}\right)$. The remarks above show that the homomorphism $\mathscr{S}_{\sigma}^{*} \rightarrow \operatorname{Aut}_{s}(\mathscr{G}(\hat{\sigma}))$ has image precisely the geometric automorphism group. Hence our construction of generators for $\mathscr{S}_{\sigma}^{*}$ gives generators for the geometric automorphism group. (It remains an open question whether every special automorphism is geometric.) 


\section{REFERENCES}

[A] E. Artin, The theory of braids, Ann. of Math. 48 (1947), 101-126.

[Ba] G. Baumslag, Lecture notes on nilpotent groups, CBMS Regional Conf. Ser. in Math., vol. 2, Amer. Math. Soc., Providence, RI, 1971.

[B] J. Birman, Braids, links and mapping class groups, Ann. of Math. Stud., vol. 82, Princeton Univ. Press, Princeton, NJ, 1974.

[BM] J. Birman and W. Menasco, (1) Studying links via closed braids, (2) Studying links via closed braids II: Classifying links which are closed 3-braids, (3) Closed braid representatives of split links and composite links, (4) Closed braid representatives of the unlink, preprints, Columbia Univ., 1988.

[C] T. Cochran, Derivatives of links: Milnor's concordance invariants and Massey's products, Mem. Amer. Math. Soc., vol. 84, Amer. Math. Soc., Providence, RI, 1990..

[D] J.-Y. le Dimet, Cobordisme d'enlacements de disques, Mém. Soc. Math. France, no. 32, Supplément Bull. Soc. Math. France 116 (1988).

[F] R. Fenn (ed.), Low dimensional topology, London Math. Soc. Lecture Note Ser., vol. 95, Cambridge Univ. Press, London, 1985.

[G1] D. Goldsmith, Homotopy of links: in answer to a question of E. Artin, Topology Conference, Lecture Notes in Math., vol 357, Springer-Verlag, Berlin, Heidelberg, New York, 1974, pp. 91-96.

[G2] Concordance implies homotopy for classical links in $M^{3}$, Comment. Math. Helv. 54 (1979), 347-355.

[Gi] C. Giffen, Link concordance implies link homotopy, Math. Scand. 45 (1979), 243-254.

$[\mathrm{HL}]$ N. Habegger and X.-S. Lin, On Milnor's $\bar{\mu}$-invariants and concordance classification of links, (in preparation).

[J] V. Jones, A polynomial invariant for links via von Neuman algebras, Bull. Amer. Math. Soc. 12 (1985), 103-111.

[Le1] J. Levine, Surgery equivalence of links, Topology 26 (1987), 45-61.

[Le2] An approach to homotopy classification of links, Trans. Amer. Math. Soc. 306 (1988), 361-387.

[L] X.-S. Lin, Artin-type representation theorems and Milnor's $\bar{\mu}$-invariants, Ph.D. thesis, Univ. of California, San Diego, 1988.

[Ma] W. Massey, Higher order linking numbers, Proc. Conference on Algebraic Topology, Univ. of Illinois, Chicago Circle, 1968, pp. 174-205.

[M1] J. Milnor, Link groups, Ann. of Math. 59 (1954), 177-195.

[M2] _ Isotopy of links, Algebraic Geometry and Topology, Princeton Univ. Press, Princeton, NJ, 1957.

[MKS] W. Magnus, A. Karrass, and D. Solitar, Combinatorial group theory, Pure and Appl. Math., vol. XIII, Interscience, NY, 1966.

[O] K. Orr, Homotopy invariants of links, Invent. Math. 95 (1989), 379-394.

[P] R. Porter, Milnor's $\bar{\mu}$-invariants and Massey products, Trans. Amer. Math. Soc. 257 (1980), 39-71.

[S] J. Stallings, Homology and central series of groups, J. Algebra 20 (1965), 170-181.

[T] V. G. Turaev, The Milnor invariants and Massey products, J. Soviet Math. 66 (1976), 189-203.

[W] E. Witten, Quantum field theory and the Jones polynomial, preprint, Institute for Advanced Study, Princeton, NJ, 1988.

Department of Mathematics, University of Georgia, Athens, Georgia 30602

Department of Mathematics, Columbia University New York, New York 10027 